\title{
Power Allocation for Robust Distributed Best-Linear-Unbiased Estimation Against Sensing Noise Variance Uncertainty
}

\author{
Jwo-Yuh Wu, Member, IEEE. and Tsang-Yi Wang, Member, IEEE
}

\begin{abstract}
Motivated by the fact that system parameter mismatch occurs in real-world sensing environments, this paper proposes power allocation schemes for robust distributed bestlinear-unbiased estimation (BLUE) that take account of the uncertainty in the local sensing noise levels. Assuming that (i) the sensing noise variance follows a statistical distribution widely used in the literature and (ii) the link channel gains between sensor nodes and the fusion center (FC) are i.i.d. Rayleigh fading, we propose to use the average reciprocal mean square error (ARMSE), averaged with respect to the distributions of sensing noise variance and fading channels, as the distortion measure. A fundamental inequality characterizing the relation between ARMSE and the average mean square error (AMSE) is established to justify the proposed design metric. While the exact formula for ARMSE is difficult to find, we derive an associated closed-form lower bound which involves the incomplete gamma function. To further ease analysis, we further derive a key inequality that specifies the range of the ARMSE lower bound. Particularly, it is shown that the boundary points of this inequality are characterized by a common function, which involves the Gaussian-tail $Q(\cdot)$ and is thus more analytically appealing. By conducting optimization on the basis of such a function, we obtain closed-form robust solutions for two power allocation problems: (i) optimizing distortion metric under a total power constraint, and (ii) minimizing total power under a target distortion requirement. In case that instantaneous channel state information (CSI) is available to the $\mathrm{FC}$, the proposed approach can be easily modified to derive analytic robust power allocation factors best matched to the CSI realizations. Computer simulations evidence the effectiveness of the proposed schemes.
\end{abstract}

Index Terms-Sensor networks, distributed estimation, best linear unbiased estimation, robustness, power allocation.

\section{INTRODUCTION}

D ISTRIBUTED estimation using wireless sensor networks is well suited for many situation awareness applications, such as environmental monitoring, positioning and tracking, temperature control, and military surveillance, to name just a

Manuscript received August 7, 2012; revised January 16 and March 12, 2013; accepted March 16, 2013. The associate editor coordinating the review of this paper and approving it for publication was S. Affes.

J.-Y. Wu is with the Department of Electrical and Computer Engineering, National Chiao Tung University, Taiwan (e-mail: jywu@cc.nctu.edu.tw).

T.-Y. Wang is with the Institute of Communications Engineering, National Sun Yat-sen University, Taiwan (e-mail: tcwang@ @aculty.nsysu.edu.tw).

This work was supported in part by the NSC of Taiwan, R.O.C., under grants NSC 100-2221-E-009-104-MY3, NSC 101-2221-E-110-068, by the Ministry of Education of Taiwan under the MoE ATU Program, and by the Telecommunication Laboratories, Chunghwa Telecom Co., Ltd. under grant TL-101-G106.

Digital Object Identifier 10.1109/TCOMM.2013.050613.121161 few [1]-[5]. A widely considered system architecture for distributed estimation consists of a set of spatially deployed sensor nodes which communicate over non-ideal wireless channels with a remotely located fusion center (FC) for retrieving the unknown deterministic parameter of interest. The design of distributed estimation algorithms in such a scenario is commonly based on either the maximum likelihood (ML) principle [6]-[12] or the best linear unbiased estimation (BLUE) scheme [13]-[23], depending on the available statistical characterization of the local sensing noise. While the ML approaches can in general yield a better performance, they require the exact knowledge of the probability density function of the sensing noise which in certain harsh application environments is rather difficult (or almost impossible) to acquire. Moreover, the ML solution is typically obtained via computationally-intensive numerical optimization. By contrast, the BLUE based schemes require only the first and second moments of all background noise, and are thus more attractive from an implementation point of view. Moreover, BLUE based fusion rules can benefit from the linearity nature and are more easily scalable with the network size as compared with the ML methods. Given these advantages, distributed BLUE thus attracted considerable attention in the past few years. To meet the critical demand of power/energy efficiency in wireless sensor networks, most of the existing BLUE proposals dealt with power allocation and minimization in order to achieve the utmost estimation accuracy [13]-[16], [21]-[23]. The development of power scheduling schemes therein typically assumed that the local sensing noise variance and the instantaneous channel state information (CSI) are exactly known at the FC.

In real-world sensing environments, uncertainty in the system parameters, such as variation in the sensing noise level caused by the change of environmental conditions or malfunction of sensor nodes, is unavoidable. Hence, in addition to energy efficiency and scalability, robustness against the system parameter mismatch is also a crucial requirement in the design of distributed estimation algorithms. Even though there have been several works about robust distributed estimation via sensor networks [24]-[26], related study in the context of the distributed BLUE, however, remains lacking. In this paper we consider a wireless sensor network employing the amplify-and-forward protocol (as in [16], [22], [23]), and propose a robust distributed BLUE scheme that takes into account the uncertain nature of the local sensing noise power. 
We adopt the Bayesian formulation, and assume that ${ }^{1}$ (i) the sensing noise variance follows the distribution as considered in [12]-[14], [18], [19], [21]-[23]; (ii) the fading channels are Rayleigh distributed, as commonly assumed in many studies of channel-aware distributed signal processing schemes [27][29]. The proposed design aims at reducing the mean square error (MSE) averaged with respect to the distributions of both the sensing noise variance and fading channels, hereafter referred to as average mean square error (AMSE). The main technical contributions of this paper can be summarized as follows.

(I) While the AMSE is not analytically tractable, we propose to adopt the reciprocal MSE averaged over the considered distributions, termed as the averaged reciprocal MSE (ARMSE) in the sequel, as the performance measure ${ }^{2}$. A fundamental relation between the ARMSE and the exact AMSE is analytically characterized. The result asserts that, toward AMSE reduction, a justified approach is through ARMSE maximization. This forms the foundation for the design principle behind the proposed robust distributed BLUE.

(II) The exact formula of ARMSE remains difficult to find. To resolve this difficulty, we first derive an associated analytic lower bound that involves the complicated incomplete Gamma function [31]. To further ease analysis, a key inequality that characterizes a feasible range of the derived ARMSE lower bound is established. The crucial fact of our findings is that the two boundary points of the inequality are entirely characterized by a common function, which involves the Gaussiantail $Q(\cdot)$ function and, thus, is more analytically appealing. Hence, instead of directly maximizing the ARMSE or its lower bound, we propose to conduct maximization with respect to this function so as to facilitate an analytically feasible (though suboptimal) sensor power allocation scheme. It is noted that, toward analytic tractability, problem formulations and system designs based on optimization of certain lower/upper bounds of the performance measures have been widely adopted in the literature of distributed estimation. For example, in [23], the authors relied on maximization of a lower bound of the objective function for MSE reduction; also, in [32], [33], minimization of certain upper bounds of MSE was considered.

(III) On the basis of the aforementioned approach, two sensor power allocation problems are addressed: (i) estimation performance enhancement under a total power constraint, and (ii) conservation of the total power budget subject to a target distortion requirement. By exploiting some attractive properties of the $Q(\cdot)$ function, sensor power allocation can be formulated as convex/concave optimization problems. Even though efficient numerical algorithms are readily available for computing the solutions, as in previous works [13], [14], [18], [19], [22], [23] we instead resort to the Lagrange-multiplier technique for deriving closed-form solutions.

(IV) In case that the instantaneous channel state information

\footnotetext{
${ }^{1}$ The conventional BLUE requires only the knowledge of the first and second moments of the noise power [13]-[23]. As in most of the robust designs under parameter mismatch, extra assumptions about the model uncertainty are needed. Notably, in the deterministic setting, estimation fusion based on the min-max MSE criterion has been reported in [26], wherein the worst-case level of perturbation is assumed to be known.

${ }^{2}$ Adoption of the reciprocal of MSE as the performance metric has been found in, e.g., [30].
}

(CSI) is available to the $\mathrm{FC}$, the proposed approach can be directly modified to exploit such advantage in order to obtain robust sensor power allocation factors best matched to the instantaneous CSI. The design criterion in this case is the ARMSE conditioned on the channel realizations. Two analogous power allocation problems are addressed. The optimization problems can be directly formulated in the form of convex optimization and are then analytically solved.

(V) Our analytic study thus provides two sets of robust designs, one based on statistical CSI (or ARMSE lower bound maximization) and the other by means of instantaneous CSI (or conditional ARMSE lower bound maximization). The analytic results allow us to investigate the distinctive features of the proposed schemes.

The rest of this paper is organized as follows. Section II is the problem statement. Section III introduces the proposed ARMSE performance measure and establishes the fundamental relation between ARMSE and the true AMSE. Section IV first derives a closed-form ARMSE lower bound, based on which a tractable cost function for robust power allocation is then proposed. Section V addresses the considered sensor power allocation problem based on the objective function shown in Section IV. Section VI focuses on the case in which the instantaneous CSI is available at the FC, proposes the conditional ARMSE as the performance measure, and then studies the power allocation problems. Section VII shows the simulation results. Finally, Section VIII concludes the paper. To ease reading, most of the mathematical proofs of key theorems and lemmas are relegated to appendix.

\section{Problem Statement}

We consider a wireless sensor network, in which $N$ sensors cooperate with a FC for estimating an unknown deterministic parameter $\theta \in \mathbb{R}$. The local observation at the $i$ th sensor node is

$$
x_{i}=\theta+n_{i}, 1 \leq i \leq N
$$

where $n_{i} \in \mathbb{R}$ is a zero-mean measurement noise with variance $\sigma_{n_{i}}^{2}$. As in [16], [22], [23], we assume that the local measurements $x_{i}$ 's are transmitted over $N$ parallel flat-fading channels to the FC via the amplify-and-forward protocol. The received signal from the $i$ th sensor can thus be described as

$$
\begin{aligned}
y_{i} & =h_{i} p_{i} x_{i}+v_{i}=h_{i} p_{i}\left(\theta+n_{i}\right)+v_{i} \\
& =h_{i} p_{i} \theta+h_{i} p_{i} n_{i}+v_{i}, \quad 1 \leq i \leq N,
\end{aligned}
$$

where $h_{i} \in \mathbb{R}$ is channel gain, $p_{i}$ is the power amplification factor for the $i$ th sensor, and $v_{i} \in \mathbb{R}$ is the zero-mean white noise with variance $\sigma_{v_{i}}^{2}$. Throughout the paper we shall set $\sigma_{v_{i}}^{2}=\sigma_{v}^{2}$ for all $1 \leq i \leq N$. Based on the received data $y_{i}$ 's in (2) and assuming that the sensing noise $n_{i}$ 's are i.i.d. and are independent of the channel noise, the parameter $\theta$ is estimated at the FC via the BLUE principle [34] as

$$
\hat{\theta}=\left[\sum_{i=1}^{N} \frac{p_{i} h_{i} y_{i}}{p_{i}^{2} h_{i}^{2} \sigma_{n_{i}}^{2}+\sigma_{v}^{2}}\right]\left[\sum_{i=1}^{N} \frac{1}{\sigma_{n_{i}}^{2}+\sigma_{v}^{2} /\left(p_{i}^{2} h_{i}^{2}\right)}\right]^{-1} .
$$


The incurred MSE of the estimate (3) is given by [34]

$$
M S E=\left[\sum_{i=1}^{N} \frac{1}{\sigma_{n_{i}}^{2}+\sigma_{v}^{2} /\left(p_{i}^{2} h_{i}^{2}\right)}\right]^{-1} .
$$

Even though one may have a nominal measurement of the local sensing noise power $\sigma_{n_{i}}^{2}$, the true noise condition could be even more worse due to, e.g., gradual drainage of battery power or sensor failures. Once a nominal noise power threshold, say, $\delta_{i}$, is determined for the $i$ th sensor, we shall seek for a statistical characterization of the uncertain noise power degradation from $\delta_{i}$. Motivated by [12]-[14], [18], [19], [21], [22], a simple yet intuitive model to pin down such uncertainty is $\sigma_{n_{i}}^{2}=\delta_{i}+z_{i}, 1 \leq i \leq N$, where $z_{i} \sim \chi_{1}^{2}$, the central ChiSquare random variable of degrees-of-freedom equal to one [35]. To further take into consideration that the severity of uncertainty could be different from sensor to sensor, in this paper we thus stick to the following assumption about the local sensing noise variance.

Assumption (A1): The sensing noise variances $\sigma_{n_{i}}^{2}$ 's obey the following statistical model

$$
\sigma_{n_{i}}^{2}=\delta_{i}+\alpha_{i} z_{i}, 1 \leq i \leq N
$$

where $\delta_{i}$ is the nominal noise variance at the $i$ th node, $z_{i} \sim \chi_{1}^{2}$ is the central Chi-Square random variable with degrees-offreedom equal to one (assumed to be i.i.d. over $i$ ), and $\alpha_{i}$ reflects the severity of uncertainty of the $i$ th sensor node.

Regarding the distribution of the fading channels, we adopt the common assumption made in the literature, e.g., [27]-[29].

Assumption (A2): The channel gains $h_{i}$ 's are i.i.d. Rayleigh distributed.

The goal of this paper is to design the sensor power allocation factors $p_{i}$ 's so as to improve the average MSE performance, taking into account the distributions of the sensing noise variance and fading channels.

\section{Proposed Design CRITERION}

\section{A. Performance Measure: ARMSE}

Given the statistical models of the sensing noise variance and the fading channels, one widely-used performance measure in the Bayesian setting is the AMSE, which by invoking (4) and (5) reads

$$
\begin{aligned}
& E\left\{\left[\sum_{i=1}^{N} \frac{1}{\sigma_{n_{i}}^{2}+\sigma_{v}^{2} /\left(p_{i}^{2} h_{i}^{2}\right)}\right]^{-1}\right\} \\
& =\int_{\mathbf{h}} \int_{\mathbf{z}}\left[\sum_{i=1}^{N} \frac{1}{\delta_{i}+\alpha_{i} z_{i}+\sigma_{v}^{2} /\left(p_{i}^{2} h_{i}^{2}\right)}\right]^{-1} f_{\mathbf{Z}}(\mathbf{z}) f_{\mathbf{H}}(\mathbf{h}) d \mathbf{z} d \mathbf{h},
\end{aligned}
$$

where $\mathbf{z} \triangleq\left[z_{1} \cdots z_{N}\right]^{T}$ and $\mathbf{h} \triangleq\left[h_{1}^{2} \cdots h_{N}^{2}\right]^{T}$, with $f_{\mathbf{Z}}(\mathbf{z})$ and $f_{\mathbf{H}}(\mathbf{h})$ being the associated distributions. However, the integrand in (6) involves an "inverse operation," which makes it quite difficult, if not impossible, to derive an analytic expression for AMSE based on (6). As a result, the design of the optimal power allocation factors based on minimization of AMSE is intractable. This thus motivates us to seek for alternative performance measures which can not only reflect the true AMSE tendency but also facilitate an analytically tractable problem formulation. In this paper, we propose to instead adopt the ARMSE, defined to be

$$
D \triangleq \int_{\mathbf{h}} \int_{\mathbf{z}} \sum_{i=1}^{N} \frac{1}{\delta_{i}+\alpha_{i} z_{i}+\sigma_{v}^{2} /\left(p_{i}^{2} h_{i}^{2}\right)} f_{\mathbf{Z}}(\mathbf{z}) f_{\mathbf{H}}(\mathbf{h}) d \mathbf{z} d \mathbf{h},
$$

as the performance metric. From an analytic perspective, the ARMSE is more appealing than AMSE since the integrand in (7) no longer involves the inverse operation; as will be shown later, this attractive feature does allow for an analytic study of the power allocation problem. Before formally introducing the proposed problem formulation on the basis of ARMSE, let us first provide a more precise mathematical connection between ARMSE and AMSE; this will further justify the adoption of the ARMSE as the performance measure in the considered problem. Toward this end, let us assume that a target performance constraint in terms of AMSE is given by

$$
E\left\{\left[\sum_{i=1}^{N} \frac{1}{\sigma_{n_{i}}^{2}+\sigma_{v}^{2} /\left(p_{i}^{2} h_{i}^{2}\right)}\right]^{-1}\right\} \leq \varepsilon,
$$

where $\varepsilon$ is a tolerable distortion level. Based on (8), we have the following proposition.

Proposition 3.1: Let $D$ be the ARMSE defined in (7). Assume that the AMSE satisfies (8). The following inequality holds:

$$
D^{-1} \leq E\left\{\left[\sum_{i=1}^{N} \frac{1}{\sigma_{n_{i}}^{2}+\sigma_{v}^{2} /\left(p_{i}^{2} h_{i}^{2}\right)}\right]^{-1}\right\} \leq \varepsilon+D^{-1} .
$$

\section{[Proof]: See Appendix A.}

Inequality (9) asserts that the AMSE, while lower bounded by $D^{-1}$, does not exceed the sum of $D^{-1}$ and the target distortion level $\varepsilon$. In particular, if $\varepsilon$ is set to be fairly small so as to guarantee satisfactory parameter estimation quality, $D^{-1}$ will then be a good approximation of AMSE. This fact asserts that, toward AMSE reduction, one can focus on simply keeping $D^{-1}$ small, or equivalently, making $D$ large. Hence, rather than direct minimization of AMSE, we will focus on maximization of ARMSE for sensor power allocation.

\section{B. Achievable AMSE}

The proposed design criterion, namely, maximization of ARMSE, hinges on a potentially small AMSE level $\varepsilon$ to guarantee that $D^{-1}$ is close to the true AMSE. In what follows we will show that, under a total transmit power constraint, $\varepsilon$ can indeed remain small provided that $N$, the total number of sensor nodes, is large enough. This thus justifies the proposed approach. To see this, we first use the Cauchy-Schwartz inequality to get

$$
\left[\sum_{i=1}^{N} \frac{1}{\sigma_{n_{i}}^{2}+\sigma_{v}^{2} /\left(p_{i}^{2} h_{i}^{2}\right)}\right]\left[\sum_{i=1}^{N} \sigma_{n_{i}}^{2}+\sigma_{v}^{2} /\left(p_{i}^{2} h_{i}^{2}\right)\right] \geq N^{2},
$$


or equivalently,

$$
\begin{aligned}
& {\left[\sum_{i=1}^{N} \frac{1}{\sigma_{n_{i}}^{2}+\sigma_{v}^{2} /\left(p_{i}^{2} h_{i}^{2}\right)}\right]^{-1}} \\
& \quad \leq \frac{1}{N^{2}}\left[\sum_{i=1}^{N} \sigma_{n_{i}}^{2}+\sigma_{v}^{2} /\left(p_{i}^{2} h_{i}^{2}\right)\right] \\
& \quad \stackrel{(a)}{=} \frac{1}{N^{2}}\left[\sum_{i=1}^{N} \delta_{i}+\alpha_{i} z_{i}+\sigma_{v}^{2} /\left(p_{i}^{2} h_{i}^{2}\right)\right],
\end{aligned}
$$

where (a) follows from (5). Based on (11), we derive in the following lemma an upper bound on AMSE.

Lemma 3.2: Under the Assumptions (A.1) and (A.2), the AMSE is upper bounded as

$$
\begin{aligned}
& E\left\{\left[\sum_{i=1}^{N} \frac{1}{\sigma_{n_{i}}^{2}+\sigma_{v}^{2} /\left(p_{i}^{2} h_{i}^{2}\right)}\right]^{-1}\right\} \\
& \leq \frac{1}{N^{2}}\left\{\sum_{i=1}^{N}\left(\delta_{i}+\alpha_{i}\right)+\frac{\sigma_{v}^{2} \sqrt{2 \pi}}{2} \sum_{i=1}^{N} \frac{1}{p_{i}^{2}}\right\} .
\end{aligned}
$$

[Proof]: See Appendix B.

Now, let $\bar{\delta}+\bar{\alpha}=\max _{i}\left(\delta_{i}+\alpha_{i}\right)$ and with $p_{i}^{2}=1$ for all $1 \leq i \leq N$, inequality (12) reads

$E\left\{\left[\sum_{i=1}^{N} \frac{1}{\sigma_{n_{i}}^{2}+\sigma_{v}^{2} /\left(p_{i}^{2} h_{i}^{2}\right)}\right]^{-1}\right\} \leq \frac{1}{N}\left\{\bar{\delta}+\bar{\alpha}+\frac{\sigma_{v}^{2} \sqrt{2 \pi}}{2}\right\}$.

Hence, for small $\bar{\delta}, \bar{\alpha}$, and $\sigma_{v}^{2}$, the upper bound in (13) can be kept small for a sufficiently large $N$, even with mere a suboptimal uniform power allocation scheme. The above facts assert that it is indeed plausible to impose a small distortion level $\varepsilon$ on the AMSE as in (8), and then rely on maximization of the ARMSE for sensor power allocation. Hence, the design paradigm can be totally shifted from AMSE minimization to ARMSE maximization.

\section{ANALYTIC LOWER BOUNDS FOR ARMSE \& Tractable Performance Metric}

To maximize the ARMSE, a conceivable approach would be to first find an analytic formula for $D$ in (7). This, however, turns out to be intractable since the double integral involved in (7) does not directly lead to a closed-form expression. To overcome this difficulty, a commonly used design strategy (though suboptimal) is to seek for a tractable lower bound for the ARMSE, and then conduct maximization with respect to this lower bound. In light of this point, we shall first derive a closed-form lower bound for ARMSE in Section IVA. As we will see, this lower bound, however, involves the incomplete gamma function and, thus, remains formidable to be analytically tackled. Based on further analyses, Section IV$\mathrm{B}$ then derives a key inequality which characterizes a plausible range for the ARMSE lower bound. The significance of the result lies in that the two boundary points of the inequality are completely specified by a common function, which is more analytically appealing than the ARMSE lower bound and, thus, can serve as the objective function for an analytic design of power allocation factors. The sensor power allocation problems based on such a performance metric will be addressed in Section V.

\section{A. Lower Bound for ARMSE}

Let us first focus on the inner integral on the left-hand side of (7). For a fixed $\mathbf{h} \triangleq\left[h_{1}^{2} \cdots h_{N}^{2}\right]^{T}$, we have

$$
\begin{aligned}
& \int_{\mathbf{z}} \sum_{i=1}^{N} \frac{1}{\delta_{i}+\alpha_{i} z_{i}+\sigma_{v}^{2} /\left(p_{i}^{2} h_{i}^{2}\right)} f_{\mathbf{Z}}(\mathbf{z}) d \mathbf{z} \\
& =\frac{1}{\sqrt{2 \pi}} \sum_{i=1}^{N} \int_{0}^{\infty} \frac{e^{-z_{i} / 2}}{\left[\delta_{i}+\alpha_{i} z_{i}+\sigma_{v}^{2} /\left(p_{i}^{2} h_{i}^{2}\right)\right] \sqrt{z_{i}}} d z_{i},
\end{aligned}
$$

in which equality holds since $z_{i} \sim \chi_{1}^{2}$ is i.i.d. and $p_{\chi_{1}^{2}}(z)=\frac{1}{\sqrt{2 \pi z}} \exp (-z / 2) u(z)[35, \mathrm{p}-24]$, where $u(z)$ denotes the unit step function. The integral in the summand in (14) admits a closed-form formula, as established in the next lemma.

Lemma 4.1: The following result holds:

$$
\begin{aligned}
& \int_{0}^{\infty} \frac{e^{-z_{i} / 2}}{\left[\delta_{i}+\alpha_{i} z_{i}+\sigma_{v}^{2} /\left(p_{i}^{2} h_{i}^{2}\right)\right] \sqrt{z_{i}}} d z_{i} \\
& =\frac{2 \pi e^{\left[\delta_{i}+\sigma_{v}^{2} /\left(p_{i}^{2} h_{i}^{2}\right)\right] / 2 \alpha_{i}} Q\left(\sqrt{\left[\delta_{i}+\sigma_{v}^{2} /\left(p_{i}^{2} h_{i}^{2}\right)\right] / \alpha_{i}}\right)}{\sqrt{\alpha_{i}\left[\delta_{i}+\sigma_{v}^{2} /\left(p_{i}^{2} h_{i}^{2}\right)\right]}},
\end{aligned}
$$

where $Q(x) \triangleq \int_{x}^{\infty} \frac{e^{-t^{2} / 2}}{\sqrt{2 \pi}} d t$ is the Gaussian tail function.

[Proof]: The proof can be done by following the similar procedures as in Appendix A in [18].

With the aid of Lemma 4.1, the average distortion measure $D$ in (7) becomes

$$
\begin{aligned}
D & =\sqrt{2 \pi} \int_{\mathbf{h}}\left\{\sum_{i=1}^{N}\right. \\
& \left.\frac{e^{\left[\delta_{i}+\sigma_{v}^{2} /\left(p_{i}^{2} h_{i}^{2}\right)\right] / 2 \alpha_{i}} Q\left(\sqrt{\left[\delta_{i}+\sigma_{v}^{2} /\left(p_{i}^{2} h_{i}^{2}\right)\right] / \alpha_{i}}\right)}{\sqrt{\alpha_{i}\left[\delta_{i}+\sigma_{v}^{2} /\left(p_{i}^{2} h_{i}^{2}\right)\right]}}\right\} f_{\mathbf{H}}(\mathbf{h}) d \mathbf{h} \\
& =\sqrt{2 \pi} \sum_{i=1}^{N} \int_{0}^{\infty} \\
& \left\{\frac{e^{\left[\delta_{i}+\sigma_{v}^{2} /\left(p_{i}^{2} h_{i}^{2}\right)\right] / 2 \alpha_{i}} Q\left(\sqrt{\left[\delta_{i}+\sigma_{v}^{2} /\left(p_{i}^{2} h_{i}^{2}\right)\right] / \alpha_{i}}\right)}{\sqrt{\alpha_{i}\left[\delta_{i}+\sigma_{v}^{2} /\left(p_{i}^{2} h_{i}^{2}\right)\right]}}\right\} \frac{e^{-\frac{h_{i}^{2}}{2}}}{2} d h_{i}^{2}
\end{aligned}
$$

where the last equality follows since $h_{i}$ 's are i.i.d. Rayleigh random variables. Direct evaluation of the integral in (16) unfortunately does not lead to a closed-form expression. To overcome this difficulty and to also facilitate a tractable design procedure, we propose to instead derive a lower bound for $D$ based on (16).

For this, we leverage the following approximation of the 
$Q(\cdot)$ function $^{3}[36, \mathrm{p}-182]$ :

$$
Q(x) \approx \frac{1}{\sqrt{2 \pi}}\left[\frac{e^{-x^{2} / 2}}{\left(1-\pi^{-1}\right) x+\pi^{-1} \sqrt{x^{2}+2 \pi}}\right] .
$$

With (17), the integrand in (16) can be approximated as (18) (see the top of next page). It is further observed that

$$
\begin{aligned}
& \sqrt{\left[\delta_{i}+\sigma_{v}^{2} /\left(p_{i}^{2} h_{i}^{2}\right)\right]^{2}+2 \pi \alpha_{i}\left[\delta_{i}+\sigma_{v}^{2} /\left(p_{i}^{2} h_{i}^{2}\right)\right]} \\
& \leq \sqrt{\left[\delta_{i}+\sigma_{v}^{2} /\left(p_{i}^{2} h_{i}^{2}\right)\right]^{2}+2 \pi \alpha_{i}\left[\delta_{i}+\sigma_{v}^{2} /\left(p_{i}^{2} h_{i}^{2}\right)\right]+\left(\pi \alpha_{i}\right)^{2}} \\
& =\sqrt{\left\{\left[\delta_{i}+\sigma_{v}^{2} /\left(p_{i}^{2} h_{i}^{2}\right)\right]+\pi \alpha_{i}\right\}^{2}}=\left[\delta_{i}+\sigma_{v}^{2} /\left(p_{i}^{2} h_{i}^{2}\right)\right]+\pi \alpha_{i} .
\end{aligned}
$$

Based on (18) and (19), we have

$$
\begin{aligned}
& \frac{e^{\left[\delta_{i}+\sigma_{v}^{2} /\left(p_{i}^{2} h_{i}^{2}\right)\right] / 2 \alpha_{i}} Q\left(\sqrt{\left[\delta_{i}+\sigma_{v}^{2} /\left(p_{i}^{2} h_{i}^{2}\right)\right] / \alpha_{i}}\right)}{\sqrt{\alpha_{i}\left[\delta_{i}+\sigma_{v}^{2} /\left(p_{i}^{2} h_{i}^{2}\right)\right]}} \\
& \geq \frac{1}{\sqrt{2 \pi}\left(1-\pi^{-1}\right)\left[\delta_{i}+\frac{\sigma_{v}^{2}}{p_{i}^{2} h_{i}^{2}}\right]+\pi^{-1}\left\{\left[\delta_{i}+\frac{\sigma_{v}^{2}}{p_{i}^{2} h_{i}^{2}}\right]+\pi \alpha_{i}\right\}} \\
& =\frac{1}{\sqrt{2 \pi}\left\{\left[\delta_{i}+\sigma_{v}^{2} /\left(p_{i}^{2} h_{i}^{2}\right)\right]+\alpha_{i}\right\}}
\end{aligned}
$$

With the aid of (16) and (20), we have the following lower bound for $D$ :

$$
\begin{aligned}
D & \geq \sum_{i=1}^{N} \int_{0}^{\infty} \frac{1}{\left[\alpha_{i}+\delta_{i}+\sigma_{v}^{2} /\left(p_{i}^{2} h_{i}^{2}\right)\right]} \frac{e^{-h_{i}^{2} / 2}}{2} d h_{i}^{2} \\
& =\frac{1}{2} \sum_{i=1}^{N} \int_{0}^{\infty} \frac{h_{i}^{2} e^{-h_{i}^{2} / 2}}{\left[\left(\sigma_{v}^{2} / p_{i}^{2}\right)+\left(\alpha_{i}+\delta_{i}\right) h_{i}^{2}\right]} d h_{i}^{2} \\
& \stackrel{(a)}{=} \underbrace{\frac{1}{2} \sum_{i=1}^{N} \int_{0}^{\infty} \frac{t_{i} e^{-t_{i} / 2}}{\left[\left(\sigma_{v}^{2} / p_{i}^{2}\right)+\left(\alpha_{i}+\delta_{i}\right) t_{i}\right]} d t_{i}}_{\triangleq \underline{D}},
\end{aligned}
$$

where (a) holds through a change of the dummy variable. By using the following integral formula (see, e.g. [31, p-348])

$$
\int_{0}^{\infty} \frac{x^{v-1} e^{-\mu x}}{x+\beta} d x=\beta^{v-1} e^{\beta \mu} \Gamma(v) \Gamma(1-v, \beta \mu)
$$

where $\Gamma(v) \triangleq \int_{0}^{\infty} e^{-t} t^{v-1} d t$ is the gamma function [31, p892] and

$$
\Gamma(\beta, x) \triangleq \int_{x}^{\infty} e^{-t} t^{\beta-1} d t
$$

is the incomplete gamma function [31, p-657], the lower bound $\underline{D}$ in (21) reads

$$
\begin{aligned}
\underline{D}=\frac{1}{2} \sum_{i=1}^{N} & \frac{\sigma_{v}^{2}}{\left[p_{i}^{2}\left(\alpha_{i}+\delta_{i}\right)^{2}\right]} e^{\sigma_{v}^{2} /\left[2 p_{i}^{2}\left(\alpha_{i}+\delta_{i}\right)\right]} \\
& \times \Gamma(2) \Gamma\left(-1, \frac{\sigma_{v}^{2}}{p_{i}^{2}\left(\alpha_{i}+\delta_{i}\right)}\right) .
\end{aligned}
$$

Remark: Note that in (19) (and (20)) equality holds if and only if $\alpha_{i}=0$, i.e., there is no uncertainty in the local sensing noise variance. In such a case, the quantity $\underline{D}$ in (24) is a close approximation to the true ARMSE.

\footnotetext{
${ }^{3}$ The approximation (17) for the $Q(\cdot)$ function is pretty sharp; see Table 4.2 in [36, p183] for a comparison of (17) and $Q(x)$ at various $x$. Numerical experiments show that (17) is even more tight than the widely used approximation $Q(x) \approx(x \sqrt{2 \pi})^{-1} e^{-x^{2} / 2}$ (see [35, p-23]).
}
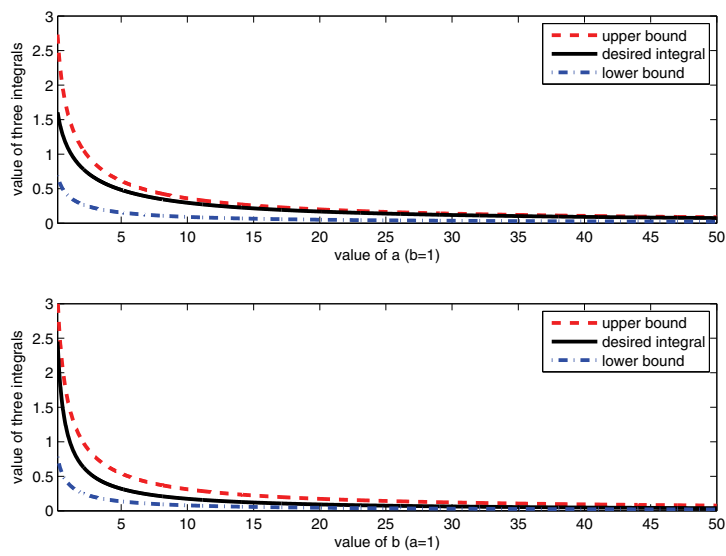

Fig. 1. Numerical verification of inequality (25) (upper bound = $\frac{6 e^{-1 / 2}}{\sqrt{8} \Gamma(3 / 2,1 / 2)} \int_{0}^{\infty} \frac{\sqrt{x} e^{-x / 2}}{a+b x} d x$, desired integral $=\int_{0}^{\infty} \frac{x e^{-x / 2}}{a+b x} d x$, lower bound $\left.=\sqrt{\frac{2-3 e^{-1 / 2}}{1-e^{-1 / 2}}} \int_{0}^{\infty} \frac{\sqrt{x} e^{-x / 2}}{a+b x} d x\right)$.

\section{B. Plausible Range of the Lower Bound $\underline{D}$}

Even though (24) admits a closed form, it nonetheless involves the incomplete gamma function and is thus difficult to analyze. To overcome this drawback, in the sequel we will further derive an inequality which specifies the plausible range of $\underline{D}$. An important feature of this result is that both the two boundary points of the inequality are entirely characterized by a common function, which involves the $Q(\cdot)$ function and is thus more analytically appealing than $\underline{D}$. To achieve this goal, we first derive the following lemma.

Lemma 4.2: For $a>0$ and $b>0$,

$$
\begin{aligned}
& \sqrt{\frac{2-3 e^{-1 / 2}}{1-e^{-1 / 2}}} \int_{0}^{\infty} \frac{\sqrt{x} e^{-x / 2}}{a+b x} d x \\
& \leq \int_{0}^{\infty} \frac{x e^{-x / 2}}{a+b x} d x \leq \frac{6 e^{-1 / 2}}{\sqrt{8} \Gamma(3 / 2,1 / 2)} \int_{0}^{\infty} \frac{\sqrt{x} e^{-x / 2}}{a+b x} d x,
\end{aligned}
$$

where $\Gamma(\beta, x)$ is the incomplete gamma function defined in (23).

[Proof]:See Appendix C.

Figure 1 numerically depicts the inequality (25); from the figure, the relationship specified by the inequality is evidenced. Based on (21) and Lemma 4.2, $\underline{D}$ can be bounded as

$$
\begin{aligned}
& \sqrt{\frac{2-3 e^{-1 / 2}}{4\left(1-e^{-1 / 2}\right)} \sum_{i=1}^{N} \int_{0}^{\infty} \frac{\sqrt{x_{i}} e^{-x_{i} / 2}}{\left(\sigma_{v}^{2} / p_{i}^{2}\right)+\left(\alpha_{i}+\delta_{i}\right) x_{i}} d x_{i}} \\
& \leq \underline{D} \leq \frac{3 e^{-1 / 2}}{\sqrt{8} \Gamma(3 / 2,1 / 2)} \sum_{i=1}^{N} \int_{0}^{\infty} \frac{\sqrt{x_{i}} e^{-x_{i} / 2}}{\left(\sigma_{v}^{2} / p_{i}^{2}\right)+\left(\alpha_{i}+\delta_{i}\right) x_{i}} d x_{i} .
\end{aligned}
$$

The integral involved in the two boundary points in (26) can be further expressed in a closed form; more precisely, we have the following lemma. 


$$
\begin{aligned}
& \frac{e^{\left[\delta_{i}+\sigma_{v}^{2} /\left(p_{i}^{2} h_{i}^{2}\right)\right] / 2 \alpha_{i}} Q\left(\sqrt{\left[\delta_{i}+\sigma_{v}^{2} /\left(p_{i}^{2} h_{i}^{2}\right)\right] / \alpha_{i}}\right)}{\sqrt{\alpha_{i}\left[\delta_{i}+\sigma_{v}^{2} /\left(p_{i}^{2} h_{i}^{2}\right)\right]}} \\
& \quad \approx \frac{1}{\sqrt{2 \pi}\left(1-\pi^{-1}\right)\left[\delta_{i}+\sigma_{v}^{2} /\left(p_{i}^{2} h_{i}^{2}\right)\right]+\pi^{-1} \sqrt{\left[\delta_{i}+\sigma_{v}^{2} /\left(p_{i}^{2} h_{i}^{2}\right)\right]^{2}+2 \pi \alpha_{i}\left[\delta_{i}+\sigma_{v}^{2} /\left(p_{i}^{2} h_{i}^{2}\right)\right]}}
\end{aligned}
$$

Lemma 4.3: For $a>0$ and $b>0$,

$$
\int_{0}^{\infty} \frac{\sqrt{x} e^{-x / 2}}{a+b x} d x=\frac{\sqrt{2 \pi}}{b}-\frac{2 \pi \sqrt{a} e^{a / 2 b}}{b \sqrt{b}} Q(\sqrt{a / b}) .
$$

[Proof]:See Appendix D.

Combining (26) and (27), we summarize the main result of this section in the following theorem.

Theorem 4.4: Let $\underline{D}$ be the ARMSE lower bound given in (24). Then the following result holds:

$$
\sqrt{\frac{2-3 e^{-1 / 2}}{4\left(1-e^{-1 / 2}\right)}} D^{\prime} \leq \underline{D} \leq \frac{3 e^{-1 / 2}}{\sqrt{8} \Gamma(3 / 2,1 / 2)} D^{\prime},
$$

where

$$
\begin{aligned}
D^{\prime} & \triangleq \sum_{i=1}^{N}\left\{\frac{\sqrt{2 \pi}}{\alpha_{i}+\delta_{i}}-\right. \\
& \left.\frac{2 \pi e^{\left\{\left(\sigma_{v}^{2} / p_{i}^{2}\right) /\left[2\left(\alpha_{i}+\delta_{i}\right)\right]\right\}} \sqrt{\left(\sigma_{v}^{2} / p_{i}^{2}\right)}}{\left(\alpha_{i}+\delta_{i}\right) \sqrt{\left(\alpha_{i}+\delta_{i}\right)}} Q\left[\sqrt{\frac{\left(\sigma_{v}^{2} / p_{i}^{2}\right)}{\alpha_{i}+\delta_{i}}}\right]\right\} .
\end{aligned}
$$

From (28), the ARMSE lower bound $\underline{D}$ is seen to be bounded from above, and below, by a scalar multiple of the common function $D^{\prime}$ (by computation, (28) reads $0.3386 D^{\prime} \leq \underline{D} \leq$ $\left.2.7479 D^{\prime}\right)$. Hence, while maximization of $\underline{D}$ has to deal with the incomplete gamma function and is intractable, we propose to conduct maximization instead based on $D^{\prime}$. The main advantage of this alternative approach is that $D^{\prime}$ in (28) now involves the $Q(\cdot)$ function. As we will see in the next section, by exploiting some attractive properties of the $Q(\cdot)$ function, the sensor power allocation problems can be formulated in the form of convex optimization that can yield closed-form solutions.

\section{SEnsor Power Allocation}

We go on to address the sensor power allocation problem for enhancing the estimation performance. For analytical tractability, the alternative performance metric $D^{\prime}$ defined in (29) will be adopted in the subsequent discussions. Section V-A considers the problem of optimizing the estimation performance under a total system power constraint. In Section V-B, the problem of minimizing the total power subject to an estimation performance requirement is addressed.

\section{A. Minimal Distortion under Power Constraint}

In terms of the alternative distortion metric $D^{\prime}$, the problem of minimizing the distortion under a total power constraint can be stated as

$$
\begin{aligned}
& \text { Maximize } \frac{1}{\sqrt{2 \pi}} \sum_{i=1}^{N}\left\{\frac{\sqrt{2 \pi}}{\alpha_{i}+\delta_{i}}-\right. \\
& \left.\frac{2 \pi e^{\left\{\left(\sigma_{v}^{2} / p_{i}^{2}\right) /\left[2\left(\alpha_{i}+\delta_{i}\right)\right]\right\}} \sqrt{\left(\sigma_{v}^{2} / p_{i}^{2}\right)}}{\left(\alpha_{i}+\delta_{i}\right) \sqrt{\left(\alpha_{i}+\delta_{i}\right)}} Q\left[\sqrt{\frac{\left(\sigma_{v}^{2} / p_{i}^{2}\right)}{\alpha_{i}+\delta_{i}}}\right]\right\},
\end{aligned}
$$

$$
\text { subject to } \sum_{i=1}^{N} p_{i}^{2} \leq P, p_{i} \geq 0,1 \leq i \leq N,
$$

where $P$ denotes the total available power budget. Since the objective function in (30) is still highly nonlinear in $p_{i}$, the problem is thus difficult to solve. Thanks to the $Q(\cdot)$ function, we can obtain the following crucial inequality to facilitate analysis:

$$
\begin{aligned}
& \frac{1}{\sqrt{2 \pi}} \sum_{i=1}^{N}\left\{\frac{\sqrt{2 \pi}}{\alpha_{i}+\delta_{i}}-\right. \\
& \left.\quad \frac{2 \pi e^{\left\{\left(\sigma_{v}^{2} / p_{i}^{2}\right) /\left[2\left(\alpha_{i}+\delta_{i}\right)\right]\right\}} \sqrt{\left(\sigma_{v}^{2} / p_{i}^{2}\right)}}{\left(\alpha_{i}+\delta_{i}\right) \sqrt{\left(\alpha_{i}+\delta_{i}\right)}} Q\left[\sqrt{\frac{\left(\sigma_{v}^{2} / p_{i}^{2}\right)}{\alpha_{i}+\delta_{i}}}\right]\right\} \\
& \stackrel{(b)}{\geq} \frac{1}{\sqrt{2 \pi}} \sum_{i=1}^{N}\left\{\frac{\sqrt{2 \pi}}{\alpha_{i}+\delta_{i}}-\frac{\pi \sqrt{\left(\sigma_{v}^{2} / p_{i}^{2}\right)}}{\left(\alpha_{i}+\delta_{i}\right) \sqrt{\left(\alpha_{i}+\delta_{i}\right)}}\right\} \\
& =\sum_{i=1}^{N}\left\{\frac{1}{\alpha_{i}+\delta_{i}}-\frac{\sigma_{v} \sqrt{\pi}}{p_{i}\left(\alpha_{i}+\delta_{i}\right) \sqrt{2\left(\alpha_{i}+\delta_{i}\right)}}\right\}
\end{aligned}
$$

where (b) follows from the well-known Chernoff bound $Q(x) \leq e^{-x^{2} / 2} / 2$. Inequality (31) provides a lower bound for the cost function in problem (30) that is now convex in terms of the power allocation factor $p_{i}$. Hence, instead of directly maximizing the cost function in (30), we propose to maximize the lower bound (31); this will allow us to derive closed-form suboptimal solution, as will be seen later. The optimization problem thus becomes

$$
\begin{aligned}
& \text { Maximize } \sum_{i=1}^{N}\left\{\frac{1}{\alpha_{i}+\delta_{i}}-\frac{\sigma_{v} \sqrt{\pi}}{p_{i}\left(\alpha_{i}+\delta_{i}\right) \sqrt{2\left(\alpha_{i}+\delta_{i}\right)}}\right\}, \\
& \text { subject to } \sum_{i=1}^{N} p_{i}^{2} \leq P, p_{i} \geq 0,1 \leq i \leq N .
\end{aligned}
$$

By means of the Lagrange multiplier technique, the solution to problem (32) can be obtained as (see Appendix E for detailed derivations)

$$
\tilde{p}_{i}=\sqrt{\frac{P}{\left(\alpha_{i}+\delta_{i}\right)\left[\sum_{i=1}^{N} \frac{1}{\alpha_{i}+\delta_{i}}\right]}}, 1 \leq i \leq N .
$$




\section{B. Minimal Power under the Distortion Constraint}

We turn to consider the problem of minimizing the total power subject to a performance requirement, namely,

$$
\text { Minimize } \sum_{i=1}^{N} p_{i}^{2}
$$

subject to

$$
\begin{aligned}
& \frac{1}{\sqrt{2 \pi}} \sum_{i=1}^{N}\left\{\frac{\sqrt{2 \pi}}{\alpha_{i}+\delta_{i}}-\right. \\
& \left.\frac{2 \pi e^{\left\{\left(\sigma_{v}^{2} / p_{i}^{2}\right) /\left[2\left(\alpha_{i}+\delta_{i}\right)\right]\right\}} \sqrt{\left(\sigma_{v}^{2} / p_{i}^{2}\right)}}{\left(\alpha_{i}+\delta_{i}\right) \sqrt{\left(\alpha_{i}+\delta_{i}\right)}} Q\left[\sqrt{\frac{\left(\sigma_{v}^{2} / p_{i}^{2}\right)}{\alpha_{i}+\delta_{i}}}\right]\right\} \\
& \geq \varepsilon^{-1}, p_{i} \geq 0,1 \leq i \leq N .
\end{aligned}
$$

To facilitate analysis we propose to replace the distortion constraint in (34) with the following one, which is expressed in terms of the lower bound (31):

$$
\sum_{i=1}^{N}\left\{\frac{1}{\alpha_{i}+\delta_{i}}-\frac{\sigma_{v} \sqrt{\pi}}{p_{i}\left(\alpha_{i}+\delta_{i}\right) \sqrt{2\left(\alpha_{i}+\delta_{i}\right)}}\right\} \geq \varepsilon^{-1} .
$$

With the aid of (35), the target estimation performance can be guaranteed and, more importantly, we can then derive a closed-form power allocation scheme. We thus consider the following optimization problem with the distortion constraint modified according to (35):

Minimize $\sum_{i=1}^{N} p_{i}^{2}$

such that $\sum_{i=1}^{N}\left\{\frac{1}{\alpha_{i}+\delta_{i}}-\frac{\sigma_{v} \sqrt{\pi}}{p_{i}\left(\alpha_{i}+\delta_{i}\right) \sqrt{2\left(\alpha_{i}+\delta_{i}\right)}}\right\} \geq \varepsilon^{-1}$, $p_{i} \geq 0,1 \leq i \leq N$.

Also based on the Lagrange multiplier technique, the solution to problem (36) can be obtained as (see Appendix E for detailed derivations)

$$
\bar{p}_{i}=\frac{\sigma_{v} \sqrt{\pi}}{\sqrt{2\left(\alpha_{i}+\delta_{i}\right)}\left[1-\left(\varepsilon \sum_{i=1}^{N} \frac{1}{\alpha_{i}+\delta_{i}}\right)^{-1}\right]} .
$$

\section{Discussions}

1) By examining the second-order derivatives of the cost functions of problems (32) and (36), it is straightforward to verify that (32) and (36) are concave/convex optimization problems. Hence, (33) and (37) are the optimal solutions to, respectively, (32) and (36).

2) Observe that both the power allocation factors (33) and (37) decrease with $\delta_{i}+\alpha_{i}$, which is exactly the average local noise power as can be seen from (5):

$$
E\left\{\sigma_{n_{i}}^{2}\right\}=E\left\{\delta_{i}+\alpha_{i} z_{i}\right\}=\delta_{i}+\alpha_{i} .
$$

Hence, either for enhancing the estimation quality under limited available power or for conserving power while meeting the desired estimation performance, sensor nodes with better local measurement quality should be allocated with more power.
3) For (37) to be valid, it should be implicitly assumed that, $\left[\varepsilon \sum_{i=1}^{N} \frac{1}{\alpha_{i}+\delta_{i}}\right]^{-1} \leq 1$, which thus imposes a threshold on the target distortion level $\varepsilon$ :

$$
\varepsilon \geq\left[\sum_{i=1}^{N} \frac{1}{\alpha_{i}+\delta_{i}}\right]^{-1}
$$

Recall from (4) that, if the communication channels are error-free, that is, $\sigma_{v}^{2}=0$ and $h_{i}=1$ for all $i$, the MSE reduces to

$$
\left[\sum_{i=1}^{N} \frac{1}{\sigma_{n_{i}}^{2}}\right]^{-1}=\left[\sum_{i=1}^{N} \frac{1}{\delta_{i}+\alpha_{i} z_{i}}\right]^{-1} .
$$

Since $\delta_{i}+\alpha_{i}=E\left\{\sigma_{n_{i}}^{2}\right\}$ (see (38)), the threshold (40) can be interpreted as the achievable MSE of the distributed BLUE by means of a "virtual" network of $N$ sensors over perfect communication links, with the local sensing noise power at the $i$ th node given by the mean value $\delta_{i}+\alpha_{i}$.

4) The proposed sensor power allocation schemes (33) and (37) are obtained by taking the sensing noise variance uncertainty model (5) into consideration. If the uncertainty factor $\alpha_{i}$ in (33) and (37) is set to be zero, then we obtain the nominal (non-robust) solutions, which are expected to yield poor performances when the true noise variance varies according to the distribution (5). This will be evidenced in the subsequent simulation study.

5) It is noted that in certain related works with amplifyand-forward protocol, e.g., [16] and [37], the total transmit power constraint used in the design formulation further includes the power dedicated by local sensor observations. Specifically, under the assumption that the parameter $\theta$ is a zero-mean random variable with known variance $\sigma_{\theta}^{2}$, the average local transmit power at the $i$ th node is $P_{i}=p_{i}^{2}\left(\sigma_{\theta}^{2}+\sigma_{n_{i}}^{2}\right)$, where $p_{i}$ is the transmit power amplification factor, and $\sigma_{n_{i}}^{2}$ is the power of the local sensing noise (see, e.g., eq. (1) in [16]); the factor $\left(\sigma_{\theta}^{2}+\sigma_{n_{i}}^{2}\right)$ thus accounts for the power of the sensor observation. The total transmit power constraint in such a scenario then reads (see, e.g., [16, p-4688])

$$
\sum_{i=1}^{N} P_{i}=\sum_{i=1}^{N} p_{i}^{2}\left(\sigma_{\theta}^{2}+\sigma_{n_{i}}^{2}\right) \leq P
$$

where $P$ denotes the total power. In our paper, we consider the scenario as in [13]-[15], wherein the unknown parameter $\theta$ is assumed to be deterministic; in this case, the local transmit power (counting the part of sensor observations) should be accordingly modified as

$$
P_{i}=p_{i}^{2}\left(\theta^{2}+\sigma_{n_{i}}^{2}\right) .
$$

However, since $\theta$ is not known, knowledge about $\theta^{2}$ is not available in the adopted deterministic setting, and this is the reason why in our problem formulation (see (30) and (34)) the total transmit power constraint does not directly include the portion of transmit power dedicated by sensor observations. We note that a similar formulation for sensor power allocation has also been used in many existing studies within the deterministic 
framework, e.g., [38], [39]. In our scenario, incorporation of the power of sensor observations into the total transmit power constraint can be done with the aid of certain priori knowledge about $\theta$. For example, if $\theta$ is known to lie within the range $[-a a]$ for some $a>0$, the average local transmit power at the $i$ th node can be upper bounded by

$$
P_{i}=p_{i}^{2}\left(\theta^{2}+\sigma_{n_{i}}^{2}\right) \leq p_{i}^{2}\left(a^{2}+\sigma_{n_{i}}^{2}\right) .
$$

With the sensing noise power $\sigma_{n_{i}}^{2}$ obeying the model (5), namely, $\sigma_{n_{i}}^{2}=\delta_{i}+\alpha_{i} z_{i}$, where $z_{i} \sim \chi_{1}^{2}$, we have $E\left\{\sigma_{n_{i}}^{2}\right\}=\delta_{i}+\alpha_{i} E\left\{z_{i}\right\}=\delta_{i}+\alpha_{i}$. Hence, the power factor $P_{i}$ in (43) further averaged over the distribution of the sensing noise variance uncertainty is upper bounded as

$$
\tilde{P}_{i}=E\left\{P_{i}\right\} \leq p_{i}^{2}\left(a^{2}+\delta_{i}+\alpha_{i}\right) \leq p_{i}^{2}\left(a^{2}+\bar{\delta}+\bar{\alpha}\right),
$$

where $\bar{\delta}+\bar{\alpha}=\max _{i}\left(\delta_{i}+\alpha_{i}\right)$. Hence, the requirement that the total average transmit power, including the power of sensor observations, cannot exceed $P$ can be guaranteed by imposing the following power constraint in terms of the upper bound in (44):

$$
\sum_{i=1}^{N} \tilde{P}_{i} \leq\left(a^{2}+\bar{\delta}+\bar{\alpha}\right) \sum_{i=1}^{N} p_{i}^{2} \leq P .
$$

In particular, the second inequality in (45) can be rewritten as

$$
\sum_{i=1}^{N} p_{i}^{2} \leq \frac{P}{\left(a^{2}+\bar{\delta}+\bar{\alpha}\right)}
$$

which admits the form of the total power constraint in Problem (30). Hence, the total power constraint in our formulation can also be directly modified (up to a constant factor) to take account of the power dedicated to sensor observations.

\section{Vi. Power Allocation Based on Instantaneous CSi}

The analyses and discussions thus far focused on the ARMSE based distortion metric which is averaged over the distributions of both the sensing noise variance uncertainty and fading channels. In case that the instantaneous CSI is known to the FC, the proposed approach can be easily modified to exploit such an advantage in order to come up with power allocations schemes best matched to the instantaneous CSI. Given a set of channel realizations $\mathbf{h}=\left[h_{1}^{2} \cdots h_{N}^{2}\right]^{T}$, the objective function considered in this case is the conditional ARMSE defined by

$$
\begin{aligned}
& D(\mathbf{h}) \triangleq \int_{\mathbf{z}} \sum_{i=1}^{N} \frac{1}{\delta_{i}+\alpha_{i} z_{i}+\sigma_{v}^{2} /\left(p_{i}^{2} h_{i}^{2}\right)} f_{\mathbf{z}}(\mathbf{z}) d \mathbf{z} \\
& \stackrel{(a)}{=} \frac{1}{\sqrt{2 \pi}} \sum_{i=1}^{N} \int_{0}^{\infty} \frac{e^{-z_{i} / 2}}{\left[\delta_{i}+\alpha_{i} z_{i}+\sigma_{v}^{2} /\left(p_{i}^{2} h_{i}^{2}\right)\right] \sqrt{z_{i}}} d z_{i} \\
& \stackrel{(b)}{=} \sqrt{2 \pi} \sum_{i=1}^{N} \frac{e^{\left[\delta_{i}+\sigma_{v}^{2} /\left(p_{i}^{2} h_{i}^{2}\right)\right] / 2 \alpha_{i}} Q\left(\sqrt{\left[\delta_{i}+\sigma_{v}^{2} /\left(p_{i}^{2} h_{i}^{2}\right)\right] / \alpha_{i}}\right)}{\sqrt{\alpha_{i}\left[\delta_{i}+\sigma_{v}^{2} /\left(p_{i}^{2} h_{i}^{2}\right)\right]}} .
\end{aligned}
$$

where, as before $\mathbf{z}=\left[z_{1} \cdots z_{N}\right]^{T}$, (a) follows directly from (14), and (b) holds as a result of Lemma 4.1. Starting from (47), we can resort to the approximation (17) of the $Q(\cdot)$ function and go through the similar procedures as in (18) (21) to obtain the following lower bound for $D(\mathbf{h})$ :

$$
\begin{aligned}
D(\mathbf{h}) & \geq \sum_{i=1}^{N} \frac{1}{\left[\delta_{i}+\alpha_{i}+\sigma_{v}^{2} /\left(p_{i}^{2} h_{i}^{2}\right)\right]} \\
& =\sum_{i=1}^{N} \frac{p_{i}^{2}}{\left(\delta_{i}+\alpha_{i}\right) p_{i}^{2}+\sigma_{v}^{2} / h_{i}^{2}}
\end{aligned}
$$

Based on (48), two sensor power allocation problems similar to Section $\mathrm{V}$ are addressed below. We note that the lower bound of $D(\mathbf{h})$ in the form (48) is convex in terms of the power allocation factors $p_{i}^{2}$; this will facilitate the derivations of closed-form optimal solutions, as will be shown later. To simplify notation, in the sequel we will denote $p_{i}^{2}$ and the reciprocal link SNR $\sigma_{v}^{2} / h_{i}^{2}$ by, respectively, $P_{i}$ and $\gamma_{i}, 1 \leq$ $i \leq N$.

\section{A. Minimal Distortion under Power Constraint}

The problem of optimizing the estimation performance under a total power constraint can be formulated as

$$
\begin{aligned}
& \operatorname{Maximize} \sum_{i=1}^{N} \frac{P_{i}}{\left(\delta_{i}+\alpha_{i}\right) P_{i}+\gamma_{i}} \\
& \text { subject to } \sum_{i=1}^{N} P_{i} \leq P, P_{i} \geq 0,1 \leq i \leq N .
\end{aligned}
$$

The solution to (49) can be obtained by means of the Lagrange multiplier technique (the details are referred to Appendix F). Specifically, let us assume without loss of generality that the reciprocal of channel SNR $\gamma_{i}=\sigma_{v}^{2} / h_{i}^{2}$, s are sorted as $\gamma_{1} \geq$ $\gamma_{2} \geq \cdots \geq \gamma_{N}$. Also let us define the function

$$
f(K) \triangleq \frac{P+\sum_{j=K}^{N} \frac{\gamma_{j}}{\left(\alpha_{j}+\delta_{j}\right)}}{\sqrt{\gamma_{K}} \sum_{j=K}^{N} \frac{\sqrt{\gamma_{j}}}{\left(\alpha_{j}+\delta_{j}\right)}}, 1 \leq K \leq N
$$

Let $1 \leq K_{1} \leq N$ be the unique integer such that $f\left(K_{1}-1\right)<$ 1 and $f\left(K_{1}\right) \geq 1$; the existence and uniqueness of such $K_{1}$ is shown in Appendix $\mathrm{F}$ (if $f(K) \geq 1$ for all $1 \leq K \leq N$, then simply set $K_{1}=1$ ). Then the optimal solution can be obtained by

$$
\begin{aligned}
& \tilde{p}_{i}^{(\mathbf{h})}= \\
& \begin{cases}0, & 1 \leq i \leq K_{1}-1, \\
\sqrt{\frac{\sqrt{\gamma_{i}}\left[P+\sum_{j=K_{1}}^{N} \frac{\gamma_{j}}{\left(\alpha_{j}+\delta_{j}\right)}\right]}{\left(\alpha_{i}+\delta_{i}\right) \sum_{j=K_{1}}^{N} \frac{\sqrt{\gamma_{j}}}{\left(\alpha_{j}+\delta_{j}\right)}}-\frac{\gamma_{i}}{\left(\alpha_{i}+\delta_{i}\right)},} & K_{1} \leq i \leq N .\end{cases}
\end{aligned}
$$




\section{B. Minimal Power under the Distortion Constraint}

The problem of minimizing the total power subject to a target AMSE constraint can be formulated as follows

Minimize $\sum_{i=1}^{N} P_{i}$

subject to $\sum_{i=1}^{N} \frac{P_{i}}{\left(\delta_{i}+\alpha_{i}\right) P_{i}+\gamma_{i}} \geq \varepsilon^{-1}, P_{i} \geq 0,1 \leq i \leq N$.

Similarly, we resort to the Lagrange multiplier technique for solving (52) (details referred to Appendix G). To find the optimal solutions, as in the previous subsection we assume without loss of generality that $\gamma_{i}$ 's are sorted as $\gamma_{1} \geq \gamma_{2} \geq \cdots \geq \gamma_{N}$, and define the function

$$
g(K) \triangleq \frac{\sum_{j=K}^{N} \frac{\sqrt{\gamma_{j}}}{\left(\alpha_{j}+\delta_{j}\right)}}{\sqrt{\gamma_{K}}\left[\sum_{j=K}^{N} \frac{1}{\left(\alpha_{j}+\delta_{j}\right)}-\varepsilon^{-1}\right]} .
$$

Let $1 \leq K_{2} \leq N$ be the unique integer such that $g\left(K_{2}-1\right)<$ 1 and $g\left(K_{2}\right) \geq 1$; the existence and uniqueness of such $K_{2}$ is shown in Appendix $\mathrm{G}$ (if $g(K) \geq 1$ for all $1 \leq K \leq N$, then simply set $K_{2}=1$ ). Then the optimal solution to (52) is given by

$$
\begin{aligned}
& \bar{p}_{i}^{(\mathbf{h})}= \\
& \begin{cases}0, & 1 \leq i \leq K_{2}-1, \\
\sqrt{\frac{\sqrt{\gamma_{i}} \sum_{j=K_{2}}^{N} \frac{\sqrt{\gamma_{j}}}{\left(\alpha_{j}+\delta_{j}\right)}}{\left(\alpha_{i}+\delta_{i}\right)\left[\sum_{j=K_{2}}^{N} \frac{\left.1 \alpha_{j}+\delta_{j}\right)}{\left(\alpha^{-1}\right.}-\varepsilon^{-1}\right.}-\frac{\gamma_{i}}{\alpha_{i}+\delta_{i}},} & K_{2} \leq i \leq N .\end{cases}
\end{aligned}
$$

\section{Discussions}

1) It is straightforward to verify that (49) and (52) are concave/convex optimization problems and, hence, (51) and (54) are the optimal solutions.

2) It is observed that both the solutions (51) and (54) turn on only the portion of sensors with small $\gamma_{i}$, or equivalently, with large channel SNR. Hence, in a power limited environment, we shall use those nodes with good link quality for MSE reduction. This can also be confirmed based on the formula of the conditional ARMSE in (48), in which a small value of $\gamma_{i}=\sigma_{v}^{2} / h_{i}^{2}$ is seen to enlarge $D(\mathbf{h})$ and thus reduces the AMSE. We note that a similar energy allocation strategy has also been found in many previous works [13], [14], [16], [18], [19].

3) From (51) and (54), it can be verified that the amount of power allocated to those active nodes decreases with $\gamma_{i}$, or equivalently, increases with the link SNR. This is also consistent with the findings in [13], [14], [16], [18], [19].

4) As can be seen, the solutions (51) and (54) designed in accordance with the conditional ARMSE (when instantaneous CSI is known) turn on only those sensors with good instantaneous link quality. This is reasonable since if some channel links are too bad, the best strategy to allocate power is to concentrate the power to those with good channel links. By contrast, the ARMSE-based power allocation schemes (33) and (37), which account for the long-term behavior of fading channels, activate all the sensor nodes for parameter estimation. That is, when distributing power to the nodes based on the channel statistics, the nodes with very poor channel statistics will still be assigned some power. This is because the random characteristics of the channel realizations. More specifically, even with very poor channel statistic, the channel could occasionally have good instantaneous link quality.

\section{Simulation Results}

This section conducts several numerical simulations to evidence the presented analyses. We consider a network of $N=250$ sensors, and the channel noise variance is set to be $\sigma_{v}^{2}=0.05$. The local sensing noise variance uncertainty parameters $\alpha_{i}$ 's are generated uniformly in the interval $\left[\begin{array}{ll}0 & \alpha\end{array}\right]$, where $\alpha>0$ is the global uncertainty factor; the noise variance thresholds $\delta_{i}$ 's are likewise drawn uniformly from $\left[\begin{array}{ll}0 & \delta\end{array}\right]$ for $\delta>0$ accounting for the maximal nominal value.

\section{A. Robust vs Non-Robust Solutions: Estimation Quality}

The first simulation compares the AMSE performances of the robust and nominal non-robust solutions designed according to different CSI knowledge. In each Monte-Carlo trial, the following two sets of schemes are implemented: (i) the robust solution (33) designed in accordance with ARMSE (7), and the associated nominal solution obtained via setting $\alpha_{i}=0$ in (33); (ii) the robust solution (51) designed based on the conditional ARMSE (47), and the associated nominal solution obtained via setting $\alpha_{i}=0$ in (51). In the sequel, we will simply call the nominal solutions the non-robust solutions; this is because they are designed using the nominal value of the noise power $\left(\sigma_{n_{i}}^{2}=\delta_{i}\right.$ since $\alpha_{i}$ is set to be zero in computing the solutions), but operate in an environment in which the true noise power deviates from $\delta_{i}$ according to the rule (5). With $\alpha=5$ and $\delta=1$, Figure 2 shows the respective simulated AMSE for different total power P. Also, with $P=40$ and $\delta=1$, Figure 3 depicts the AMSE with respect to different uncertainty factors $\alpha$. Firstly, we can see from both figures that the robust designs do yield smaller AMSE for either type of CSI knowledge. Secondly, both the robust solution (33) designed on the basis of ARMSE (7) and the robust solution (51) designed in accordance with the conditional ARMSE (47) performs almost the same, despite that the latter exploits the knowledge of instantaneous CSI in each trial. Even though the scheme (33) using only the knowledge of channel statistics appears to slightly outperform the scheme (51) using the knowledge of the channel realizations, the difference between them is virtually nil in the numerical data. A possible reason for this counter-intuitive result is that both the power allocation schemes are designed based on a lower bound of ARMSE instead of the AMSE. Since the object function used for the design (ARMSE) and the evaluated performance metric (AMSE) are different, such a phenomenon then appears. From Fig. 2, it can be further observed that the AMSE decreases with $P$. This is intuitively reasonable since 


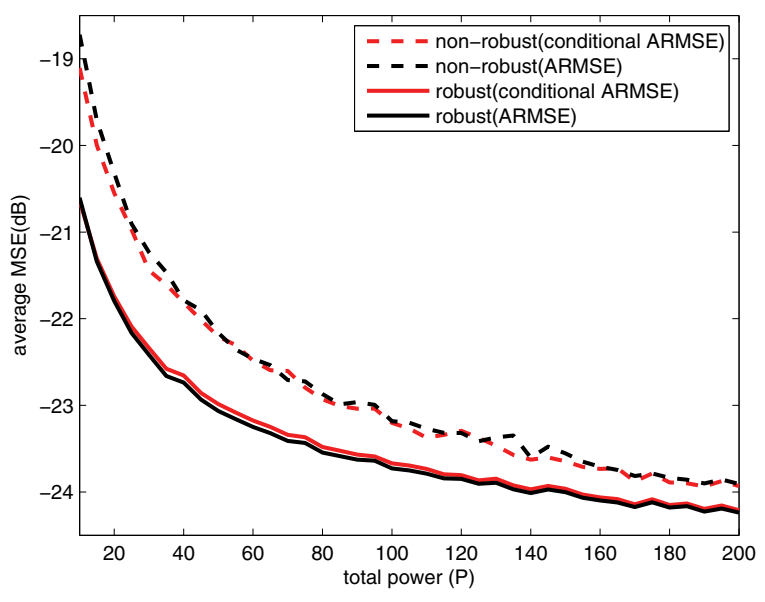

Fig. 2. Average mean square error at different total power $(\alpha=5$ and $\delta=1)$.

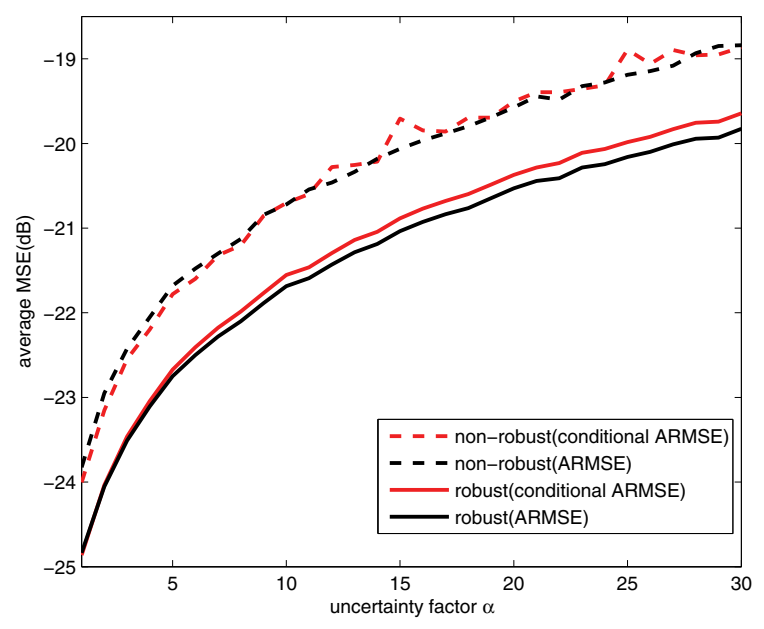

Fig. 3. Average mean square error at different uncertainty factor $\alpha(P=40$ and $\delta=1)$.

a large transmit power can enhance the overall signal quality at the FC for improving the global estimation accuracy; a similar phenomenon has also been seen in various previous works [19], [22], [23]. From Fig. 3 it is also seen that, with a fixed system power, the AMSE increases with the uncertainty factor $\alpha$. The result is not unexpected since, as $\alpha$ increases, the deviation of the true noise variance from its nominal value also increases, thereby degrading the global estimation quality.

\section{B. Robust vs Non-Robust Solutions: Power Consumption}

This simulation illustrates the power consumption of the proposed robust methods subject to the average distortion constraint. The target AMSE level is set to be $\varepsilon=0.05$ (about $-13.01 d B$ ). Figure 4(a) first compares the estimation performances of the ARMSE based robust design (37) and the corresponding non-robust counterpart. The result shows that the achievable AMSE of both schemes are below the target level. In each trial, it is further found that the minimal power attained by the robust solution (37) is smaller. To quantify the
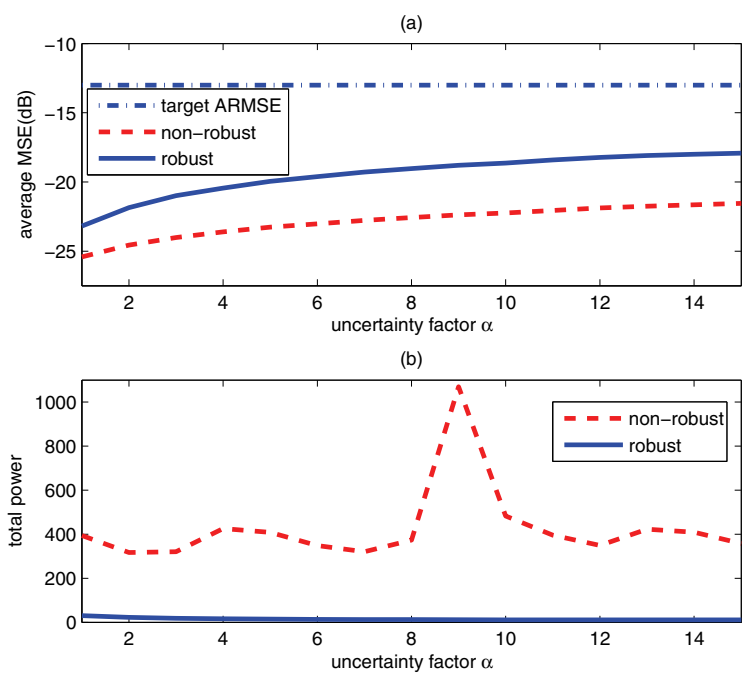

Fig. 4. Comparison of ARMSE based design (37) and the corresponding nominal no-robust solution in terms of estimation performance and power consumption $(\delta=1)$.

power consumption, Fig. 4(b) thus plots the consumed power of the two schemes. As we can see from the figure, the robust solution (37) significantly reduces the total power. Figure 5 further compares the two schemes at different target distortion levels $\varepsilon$; as expected, the robust solution (37) still greatly reduces the total power. Figure 6 depicts the performance of the conditional ARMSE based design (54). From Fig. 6(a), it can be seen that the associated nominal non-robust version even fails to keep the AMSE below the target level, although the consumed power is slightly smaller than that of the robust solution (cf. Fig. 6(b)). Hence, if the true noise variance is different from the nominal variance used in the design step, then the non-robust solution will not be "robust" in that it could violate the target performance requirement. Moreover, as $\alpha$ increases, the violation becomes more serious (note that $\alpha$ reflects the severity of uncertainty); through extensive simulations it is found that this phenomenon happens at all times. By contrast, the proposed scheme (54), which takes into consideration of the parameter uncertainty, is robust in that it always guarantees the required target performance.

\section{CONCLUSIONS}

This paper has presented an original contribution to the study of power allocation for robust distributed BLUE in the presence of sensing noise variance uncertainty. The proposed design criterion in terms of ARMSE has several unique advantages. Firstly, it is established that with a small target AMSE the inverse of ARMSE is a close approximation to the true AMSE. Given this appealing fundamental result, maximization of the ARMSE is thus a justified approach for enhancing the estimation quality. Secondly, starting from the ARMSE design metric, rigorous analyses are carried out to derive an analytically tractable objective function for the design of power allocation factors. Closed-form solutions are then derived for two typical power allocation problems. Thirdly, starting from the considered ARMSE, the proposed robust design can also be fully compatible with the case in 


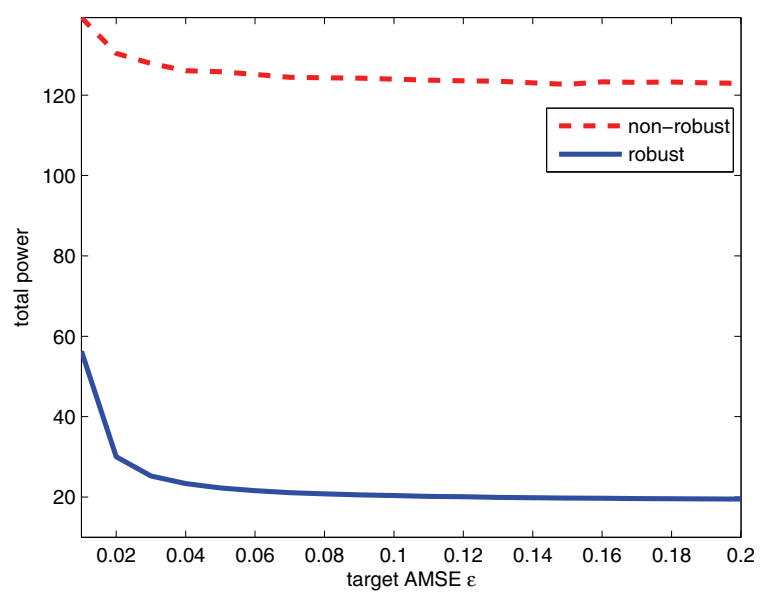

Fig. 5. Comparison of total consumed power of the robust design (37) and the corresponding nominal no-robust solution at different target distortion level $\varepsilon$ $(\delta=1)$.

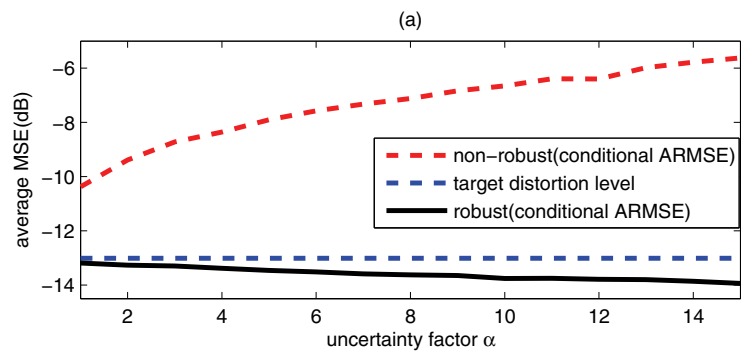

(b)

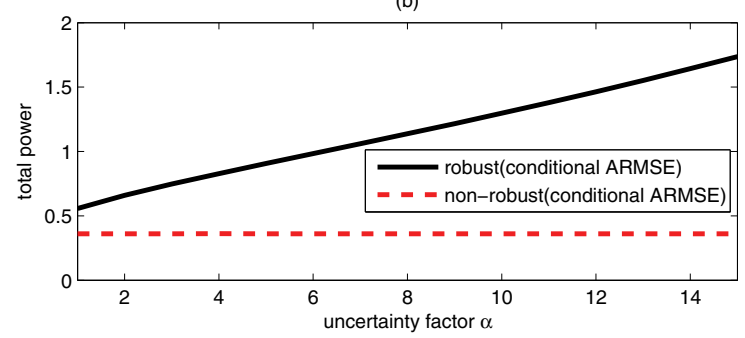

Fig. 6. Comparison of conditional ARMSE based design (54) and the corresponding nominal no-robust solution in terms of estimation performance and power consumption $(\delta=1)$.

which instantaneous CSI is available to the FC; particularly, with the conditional ARMSE naturally as the cost function, closed-form power allocation schemes best matched to the channel realizations are also obtained. In words, the proposed approach based on the ARMSE does provide a fundamental analysis framework for the study the robust distributed BLUE. Future study will go on to characterize the achievable diversity performance as well as the power scheduling gains of the proposed robust schemes. Moreover, regarding the design based CSI realizations, the issue of CSI mismatch due to imperfect training will also be further studied in the current setup.

\section{Appendix A: Proof of Proposition 3.1}

Since $D>0$, inequality (8) directly imposes the following upper bound on AMSE

$$
E\left\{\left[\sum_{i=1}^{N} \frac{1}{\sigma_{n_{i}}^{2}+\sigma_{v}^{2} /\left(p_{i}^{2} h_{i}^{2}\right)}\right]^{-1}\right\} \leq \varepsilon+D^{-1} .
$$

Now, since the function $g(x)=1 / x$ is convex for $x>0$, it follows from the Jensen's inequality [40] that

$$
\begin{aligned}
& E\left\{\left[\sum_{i=1}^{N} \frac{1}{\sigma_{n_{i}}^{2}+\sigma_{v}^{2} /\left(p_{i}^{2} h_{i}^{2}\right)}\right]^{-1}\right\} \\
& \geq\left\{E\left[\sum_{i=1}^{N} \frac{1}{\sigma_{n_{i}}^{2}+\sigma_{v}^{2} /\left(p_{i}^{2} h_{i}^{2}\right)}\right]\right\}^{-1}=D^{-1} .
\end{aligned}
$$

That is, the AMSE is lower bounded by $D^{-1}$, the reciprocal of ARMSE. Combining (A.1) and (A.2) then yields (8).

\section{Appendix B: Proof of Lemma 3.2}

From (11), we have

$$
\begin{aligned}
& E\left\{\left[\sum_{i=1}^{N} \frac{1}{\sigma_{n_{i}}^{2}+\sigma_{v}^{2} /\left(p_{i}^{2} h_{i}^{2}\right)}\right]^{-1}\right\} \\
& \leq \frac{1}{N^{2}} E\left\{\sum_{i=1}^{N} \delta_{i}+\alpha_{i} z_{i}+\sigma_{v}^{2} /\left(p_{i}^{2} h_{i}^{2}\right)\right\} \\
& \stackrel{(a)}{=} \frac{1}{N^{2}}\left\{\sum_{i=1}^{N}\left(\delta_{i}+\alpha_{i}\right)+\sigma_{v}^{2} \sum_{i=1}^{N} \frac{1}{p_{i}^{2}} E\left[\frac{1}{h_{i}^{2}}\right]\right\}
\end{aligned}
$$

where (a) holds since $E\left\{z_{i}\right\}=1$ (recalling that $z_{i} \sim \chi_{1}^{2}$ and using [35, p-25]). Hence, it suffices to derive $E\left[\frac{1}{h_{i}^{2}}\right]$, given that $h_{i}^{2}$ is the standard Rayleigh distribution. To simplify notation we designate $h_{i}^{2}$ by $x$, thereby $f_{X}(x)=x e^{-x^{2} / 2} u(x)$. With $y=1 / x$ and using the technique of function of random variable (see, e.g., [36]), the distribution of $y$ can be shown to be (through some straightforward manipulations) $f_{Y}(y)=$ $y^{-3} e^{-1 / 2 y^{2}} u(y)$. As a result,

$$
\begin{aligned}
E\left[\frac{1}{h_{i}^{2}}\right] & =E\{y\}=\int_{0}^{\infty} y^{-2} e^{-1 / 2 y^{2}} d y \stackrel{(b)}{=} \int_{0}^{\infty} e^{-t^{2} / 2} d t \\
& \stackrel{(c)}{=} \sqrt{2 \pi} / 2
\end{aligned}
$$

where (b) follows through a change of variable and (c) holds by invoking the definition of the $Q(\cdot)$ function and $Q(0)=1 / 2$. The assertion follows from (B.1) and (B.2).

\section{Appendix C: PRoOf OF LEMma 4.2}

\section{A. Proof of 1st Inequality}

We first note that

$$
\int_{0}^{\infty} \frac{\sqrt{x} e^{-x / 2}}{a+b x} d x=\int_{0}^{\infty}\left[\frac{\sqrt{x} e^{-x / 4}}{\sqrt{a+b x}}\right]\left[\frac{e^{-x / 4}}{\sqrt{a+b x}}\right] d x
$$


By the Cauchy-Schwartz inequality, it follows that

$$
\begin{aligned}
& \int_{0}^{\infty} \frac{\sqrt{x} e^{-x / 2}}{a+b x} d x \\
& =\int_{0}^{\infty}\left[\frac{\sqrt{x} e^{-x / 4}}{\sqrt{a+b x}}\right]\left[\frac{e^{-x / 4}}{\sqrt{a+b x}}\right] d x \\
& \leq\left\{\int_{0}^{\infty} \frac{x e^{-x / 2}}{a+b x} d x\right\}^{1 / 2}\left\{\int_{0}^{\infty} \frac{e^{-x / 2}}{a+b x} d x\right\}^{1 / 2} .
\end{aligned}
$$

Based on (C.2) together with some manipulations, we have

$$
\begin{aligned}
& \int_{0}^{\infty} \frac{\sqrt{x} e^{-x / 2}}{a+b x} d x \\
& \leq\left\{\int_{0}^{\infty} \frac{x e^{-x / 2}}{a+b x} d x\right\}^{1 / 2}\left\{\int_{0}^{\infty} \frac{e^{-x / 2}}{a+b x} d x\right\}^{1 / 2} \\
& =\left\{\int_{0}^{\infty} \frac{x e^{-x / 2}}{a+b x} d x\right\}^{1 / 2}\left\{\int_{0}^{1} \frac{e^{-x / 2}}{a+b x} d x+\right. \\
& \left.\int_{1}^{\infty} \frac{e^{-x / 2}}{a+b x} d x\right\}^{1 / 2} \\
& \leq\left\{\int_{0}^{\infty} \frac{x e^{-x / 2}}{a+b x} d x\right\}^{1 / 2}\left\{\int_{0}^{1} \frac{e^{-x / 2}}{a+b x} d x+\right. \\
& \left.\int_{1}^{\infty} \frac{x e^{-x / 2}}{a+b x} d x\right\}^{1 / 2} \\
& =\left\{\int_{0}^{\infty} \frac{x e^{-x / 2}}{a+b x} d x\right\}^{1 / 2}\left\{\int_{0}^{1} \frac{e^{-x / 2}}{a+b x} d x+\right. \\
& \left.\int_{0}^{\infty} \frac{x e^{-x / 2}}{a+b x} d x-\int_{0}^{1} \frac{x e^{-x / 2}}{a+b x} d x\right\}^{1 / 2} \\
& =\left\{\int_{0}^{\infty} \frac{x e^{-x / 2}}{a+b x} d x\right\}^{1 / 2}\left\{\int_{0}^{\infty} \frac{x e^{-x / 2}}{a+b x} d x+\right. \\
& \left.\int_{0}^{1} \frac{(1-x) e^{-x / 2}}{a+b x} d x\right\}^{1 / 2} \text {. }
\end{aligned}
$$

To further rewrite (C.3), we need the following technical lemma.

\section{Lemma C.1:}

$$
\int_{0}^{1} \frac{(1-x) e^{-x / 2}}{a+b x} d x \leq \rho \int_{0}^{1} \frac{x e^{-x / 2}}{a+b x} d x
$$

whenever

$$
\rho \geq \frac{\left(1-e^{-1 / 2}\right)}{\left(2-3 e^{-1 / 2}\right)}-1
$$

[Proof of Lemma C.1]: Let us write

$$
\begin{aligned}
& \int_{0}^{1} \frac{\rho x e^{-x / 2}}{a+b x} d x-\int_{0}^{1} \frac{(1-x) e^{-x / 2}}{a+b x} d x \\
& =\int_{0}^{1} \frac{[(\rho+1) x-1] e^{-x / 2}}{a+b x} d x .
\end{aligned}
$$

Also, note that, since $0 \leq x \leq 1$,

$$
\begin{aligned}
& \int_{0}^{1} \frac{[(\rho+1) x-1] e^{-x / 2}}{a+b x} d x \\
& \geq \int_{0}^{1} \frac{[(\rho+1) x-1] e^{-x / 2}}{a+b} d x \\
& =\frac{1}{a+b} \int_{0}^{1}[(\rho+1) x-1] e^{-x / 2} d x .
\end{aligned}
$$

Since $a>0$ and $b>0$, it suffices to prove

$$
\int_{0}^{1}[(\rho+1) x-1] e^{-x / 2} d x>0 .
$$

if the condition on $\rho$ specified in (C.4) holds. After straightforward manipulations, we have

$$
\begin{aligned}
& \int_{0}^{1}[(\rho+1) x-1] e^{-x / 2} d x \\
& =\left(4-6 e^{-1 / 2}\right)-\frac{2}{(\rho+1)}\left(1-e^{-1 / 2}\right) .
\end{aligned}
$$

which is no less than zero whenever $\rho$ satisfies (B.4). The proof is thus completed.

Based on (C.3) and Lemma C.1, we have

$$
\begin{aligned}
& \int_{0}^{\infty} \frac{\sqrt{x} e^{-x / 2}}{a+b x} d x \\
& \leq\left\{\int_{0}^{\infty} \frac{x e^{-x / 2}}{a+b x} d x\right\}^{1 / 2}\left\{\int_{0}^{\infty} \frac{x e^{-x / 2}}{a+b x} d x+\right. \\
& \left.\int_{0}^{1} \frac{(1-x) e^{-x / 2}}{a+b x} d x\right\}^{1 / 2} \\
& \leq\left\{\int_{0}^{\infty} \frac{x e^{-x / 2}}{a+b x} d x\right\}^{1 / 2}\left\{\int_{0}^{\infty} \frac{x e^{-x / 2}}{a+b x} d x+\right. \\
& \left.\left[\frac{\left(1-e^{-1 / 2}\right)}{\left(2-3 e^{-1 / 2}\right)}-1\right] \int_{0}^{1} \frac{x e^{-x / 2}}{a+b x} d x\right\}^{1 / 2} \\
& \leq\left\{\int_{0}^{\infty} \frac{x e^{-x / 2}}{a+b x} d x\right\}^{1 / 2}\left\{\int_{0}^{\infty} \frac{x e^{-x / 2}}{a+b x} d x+\right. \\
& \left.\left[\frac{\left(1-e^{-1 / 2}\right)}{\left(2-3 e^{-1 / 2}\right)}-1\right] \int_{0}^{\infty} \frac{x e^{-x / 2}}{a+b x} d x\right\}^{1 / 2} \\
& \leq\left\{\int_{0}^{\infty} \frac{x e^{-x / 2}}{a+b x} d x\right\}^{1 / 2}\left\{\frac{\left(1-e^{-1 / 2}\right)}{\left(2-3 e^{-1 / 2}\right)} \int_{0}^{\infty} \frac{x e^{-x / 2}}{a+b x} d x\right\}^{1 / 2} \\
& =\sqrt{\frac{\left(1-e^{-1 / 2}\right)}{\left(2-3 e^{-1 / 2}\right)}} \int_{0}^{\infty} \frac{x e^{-x / 2}}{a+b x} d x,
\end{aligned}
$$

which then implies

$$
\sqrt{\frac{\left(1-e^{-1 / 2}\right)}{\left(2-3 e^{-1 / 2}\right)}} \int_{0}^{\infty} \frac{\sqrt{x} e^{-x / 2}}{a+b x} d x \leq \int_{0}^{\infty} \frac{x e^{-x / 2}}{a+b x} d x .
$$

The proof of the first inequality is thus completed.

\section{B. Proof of 2 nd Inequality}

Since

$$
\int_{0}^{1} \frac{x e^{-x / 2}}{a+b x} d x \leq \int_{0}^{1} \frac{\sqrt{x} e^{-x / 2}}{a+b x} d x
$$


we can write

$$
\begin{aligned}
& \int_{0}^{\infty} \frac{x e^{-x / 2}}{a+b x} d x \\
& =\int_{0}^{1} \frac{x e^{-x / 2}}{a+b x} d x+\int_{1}^{\infty} \frac{x e^{-x / 2}}{a+b x} d x \\
& \stackrel{(a)}{\leq} \int_{0}^{1} \frac{\sqrt{x} e^{-x / 2}}{a+b x} d x+\int_{1}^{\infty} \frac{x e^{-x / 2}}{a+b x} d x \\
& \stackrel{(b)}{\leq} \int_{0}^{1} \frac{\sqrt{x} e^{-x / 2}}{a+b x} d x+\int_{1}^{\infty} \frac{\sqrt{x} e^{-x / 2}}{a+b x} d x+ \\
& =\int_{1}^{\infty} \frac{\sqrt{x}-\sqrt{x}) e^{-x / 2}}{a+b x} d x \\
& =\int_{0}^{-x / 2} d x+\int_{1}^{\infty} \frac{(x-\sqrt{x}) e^{-x / 2}}{a+b x} d x,
\end{aligned}
$$

where (a) follows from (C.9) and (b) holds through direct manipulations. The following lemma can be used for deriving an upper bound for the RHS of (C.10), and will be used in the proof.

Lemma C.2: Let $\Gamma(\beta, x) \triangleq \int_{x}^{\infty} e^{-t} t^{\beta-1} d t$ be the incomplete gamma function. Then

$$
\int_{1}^{\infty} \frac{(x-\sqrt{x}) e^{-x / 2}}{a+b x} d x \leq \eta \int_{1}^{\infty} \frac{\sqrt{x} e^{-x / 2}}{a+b x} d x
$$

whenever

$$
\eta \geq \frac{6 e^{-1 / 2}}{\sqrt{8} \Gamma(3 / 2,1 / 2)}-1
$$

[Proof of Lemma C.2]: Let us write

$$
\begin{aligned}
& \int_{1}^{\infty} \frac{(x-\sqrt{x}) e^{-x / 2}}{a+b x} d x-\eta \int_{1}^{\infty} \frac{\sqrt{x} e^{-x / 2}}{a+b x} d x \\
& =\int_{1}^{\infty} \frac{(x-\sqrt{x}-\eta \sqrt{x}) e^{-x / 2}}{a+b x} d x \\
& \stackrel{(a)}{\leq} \int_{1}^{\infty} \frac{(x-\sqrt{x}-\eta \sqrt{x}) e^{-x / 2}}{a+b} d x \\
& =\frac{1}{(a+b)}\left[\int_{1}^{\infty} x e^{-x / 2} d x-(\eta+1) \int_{1}^{\infty} \sqrt{x} e^{-x / 2} d x\right]
\end{aligned}
$$

where (a) holds since the domain of integration is $x \geq 1$. Based on (C.13), it suffices to show

$$
\int_{1}^{\infty} x e^{-x / 2} d x-(\eta+1) \int_{1}^{\infty} \sqrt{x} e^{-x / 2} d x \leq 0
$$

whenever (C.12) is true. After some straightforward manipulations, we have $\int_{1}^{\infty} x e^{-x / 2} d x=6 e^{-1 / 2}$ and $\int_{1}^{\infty} \sqrt{x} e^{-x / 2} d x=\sqrt{8} \Gamma(3 / 2,1 / 2)$, and hence (C.14) holds if (C.12) is true. The proof is completed.
By means of Lemma C.2, we can rewrite (C.10) as

$$
\begin{aligned}
& \int_{0}^{\infty} \frac{x e^{-x / 2}}{a+b x} d x \\
& \leq \int_{0}^{\infty} \frac{\sqrt{x} e^{-x / 2}}{a+b x} d x+\int_{1}^{\infty} \frac{(x-\sqrt{x}) e^{-x / 2}}{a+b x} d x \\
& \leq \int_{0}^{\infty} \frac{\sqrt{x} e^{-x / 2}}{a+b x} d x+ \\
& {\left[\frac{6 e^{-1 / 2}}{\sqrt{8} \Gamma(3 / 2,1 / 2)}-1\right] \int_{1}^{\infty} \frac{\sqrt{x} e^{-x / 2}}{a+b x} d x} \\
& \leq \int_{0}^{\infty} \frac{\sqrt{x} e^{-x / 2}}{a+b x} d x+ \\
& {\left[\frac{6 e^{-1 / 2}}{\sqrt{8} \Gamma(3 / 2,1 / 2)}-1\right] \int_{0}^{\infty} \frac{\sqrt{x} e^{-x / 2}}{a+b x} d x} \\
& =\frac{6 e^{-1 / 2}}{\sqrt{8} \Gamma(3 / 2,1 / 2)} \int_{0}^{\infty} \frac{\sqrt{x} e^{-x / 2}}{a+b x} d x \text {, }
\end{aligned}
$$

which proves the second inequality in (25).

\section{Appendix D: PROOF OF LemMa 4.4}

The following lemma will be used in the proof of Lemma 4.4.

Lemma D.1: $\int_{1}^{\infty} \frac{e^{-a t / 2 b}}{t \sqrt{t-1}} d t=2 \pi Q(\sqrt{a / b})$.

[Proof]: Let us define $t=\csc ^{2} \theta$, hence $d t=$ $-2 \csc ^{2} \theta \cot \theta d \theta$ and

$$
\begin{aligned}
& \int_{1}^{\infty} \frac{e^{-a t / 2 b}}{t \sqrt{t-1}} d t \\
& =\int_{\pi / 2}^{0} \frac{e^{-a \csc ^{2} \theta / 2 b}}{\csc ^{2} \theta \cot \theta}\left(-2 \csc ^{2} \theta \cot \theta\right) d \theta \\
& =2 \int_{0}^{\pi / 2} e^{-a / 2 b \sin ^{2} \theta} d \theta .
\end{aligned}
$$

We note that the $Q(\cdot)$ function admits the following alternative expression [41, p-71]

$$
Q(x)=\frac{1}{\pi} \int_{0}^{\pi / 2} e^{-x^{2} / 2 \sin ^{2} \theta} d \theta .
$$

The assertion follows immediately from (D.1) and (D.2).

[Proof of Lemma 4.4]: The proof is based on repeated change of variables in terms of the trigonometric functions. Firstly, let $t=a+b x$; hence we have $x=(t-a) / b, d x=d t / b$, and

$$
\begin{aligned}
& \int_{0}^{\infty} \frac{\sqrt{x} e^{-x / 2}}{a+b x} d x \\
& =\frac{1}{b} \int_{a}^{\infty} \frac{\sqrt{(t-a) / b} e^{-(t-a) / 2 b}}{t} d t \\
& =\frac{e^{a / 2 b}}{b \sqrt{b}} \int_{a}^{\infty} \frac{\sqrt{t-a} e^{-t / 2 b}}{t} d t
\end{aligned}
$$

Through further change of variable by letting $t=a \csc ^{2} \theta$, hence $t-a=a\left(\csc ^{2} \theta-1\right)=a \cot ^{2} \theta$ and $d t=$ 
$-2 a \csc ^{2} \theta \cot \theta d \theta$, we have

$$
\begin{aligned}
& \frac{e^{a / 2 b}}{b \sqrt{b}} \int_{a}^{\infty} \frac{\sqrt{t-a} e^{-t / 2 b}}{t} d t \\
& =\frac{e^{a / 2 b}}{b \sqrt{b}} \int_{\pi / 2}^{0} \frac{\sqrt{a} \cot \theta\left(-2 a \csc ^{2} \theta \cot \theta\right) e^{-a \csc ^{2} \theta / 2 b}}{a \csc ^{2} \theta} d \theta \\
& =\frac{2 \sqrt{a} e^{a / 2 b}}{b \sqrt{b}} \int_{0}^{\pi / 2} \cot ^{2} \theta e^{-a \csc ^{2} \theta / 2 b} d \theta \\
& =\frac{2 \sqrt{a}}{b \sqrt{b}} \int_{0}^{\pi / 2} \cot ^{2} \theta e^{-a\left(\csc ^{2} \theta-1\right) / 2 b} d \theta \\
& =\frac{2 \sqrt{a}}{b \sqrt{b}} \int_{0}^{\pi / 2} \cot ^{2} \theta e^{-a \cot ^{2} \theta / 2 b} d \theta
\end{aligned}
$$

Then we let $y=\cot ^{2} \varphi$, thus $d y=-2 \cot \varphi \csc ^{2} \varphi d \varphi$ and

$$
\begin{aligned}
& \frac{2 \sqrt{a}}{b \sqrt{b}} \int_{0}^{\pi / 2} \cot ^{2} \varphi e^{-a \cot ^{2} \varphi / 2 b} d \varphi \\
& =\frac{\sqrt{a}}{b \sqrt{b}} \int_{0}^{\infty} \frac{y e^{-a y / 2 b}}{(1+y) \sqrt{y}} d y \\
& =\frac{\sqrt{a}}{b \sqrt{b}} \int_{0}^{\infty} \frac{\sqrt{y} e^{-a y / 2 b}}{1+y} d y .
\end{aligned}
$$

Again letting $y=\tan ^{2} \phi$, hence $d y=2 \tan \phi \sec ^{2} \phi d \phi$, the above integral can be decomposed into

$$
\begin{aligned}
& \frac{\sqrt{a}}{b \sqrt{b}} \int_{0}^{\infty} \frac{\sqrt{y} e^{-a y / 2 b}}{1+y} d y \\
& =\frac{\sqrt{a}}{b \sqrt{b}} \int_{0}^{\pi / 2} \frac{\tan \phi e^{-a \tan ^{2} \phi / 2 b}}{\sec ^{2} \phi} 2 \tan \phi \sec ^{2} \phi d \phi \\
& =\frac{2 \sqrt{a}}{b \sqrt{b}} \int_{0}^{\pi / 2} \tan ^{2} \phi e^{-a \tan ^{2} \phi / 2 b} d \phi \\
& =\frac{2 \sqrt{a}}{b \sqrt{b}} \int_{0}^{\pi / 2}\left(\sec ^{2} \phi-1\right) e^{-a \tan ^{2} \phi / 2 b} d \phi \\
& =\frac{2 \sqrt{a}}{b \sqrt{b}} \int_{0}^{\pi / 2} \sec ^{2} \phi e^{-a \tan ^{2} \phi / 2 b} d \phi- \\
& \frac{2 \sqrt{a}}{b \sqrt{b}} \int_{0}^{\pi / 2} e^{-a \tan ^{2} \phi / 2 b} d \phi .
\end{aligned}
$$

We observe that the first term in (D.6) can be rearranged as

$$
\begin{aligned}
& \frac{2 \sqrt{a}}{b \sqrt{b}} \int_{0}^{\pi / 2} \sec ^{2} \phi e^{-a \tan ^{2} \phi / 2 b} d \phi \\
& =\frac{2 \sqrt{a}}{b \sqrt{b}} \int_{0}^{\pi / 2} e^{-a \tan ^{2} \phi / 2 b} d \tan \phi \\
& =\frac{2}{b} \int_{0}^{\pi / 2} e^{-a \tan ^{2} \phi / 2 b} d(\sqrt{a} \tan \phi / \sqrt{b}) \\
& =\frac{2}{b} \int_{0}^{\infty} e^{-u^{2} / 2} d u \\
& =\frac{2 \sqrt{2 \pi}}{b} \underbrace{\frac{1}{\sqrt{2 \pi}} \int_{0}^{\infty} e^{-u^{2} / 2} d u}_{=Q(0)=1 / 2}=\frac{\sqrt{2 \pi}}{b} .
\end{aligned}
$$

Also, if we let $z=\tan ^{2} \phi$, hence $d z=2 \tan \phi \sec ^{2} \phi d \phi=$
$2 \sqrt{z}(1+z) d \phi$, the second term in (D.6) becomes

$$
\begin{aligned}
& \frac{2 \sqrt{a}}{b \sqrt{b}} \int_{0}^{\pi / 2} e^{-a \tan ^{2} \phi / 2 b} d \phi \\
& =\frac{\sqrt{a}}{b \sqrt{b}} \int_{0}^{\infty} \frac{e^{-a z / 2 b}}{\sqrt{z}(1+z)} d z .
\end{aligned}
$$

An explicit formula for the integral in (D.8) can be readily obtained by first letting $t=z+1$, hence

$$
\begin{aligned}
& \frac{\sqrt{a}}{b \sqrt{b}} \int_{0}^{\infty} \frac{e^{-a z / 2 b}}{\sqrt{z}(1+z)} d z \\
& =\frac{\sqrt{a}}{b \sqrt{b}} \int_{1}^{\infty} \frac{e^{-a(t-1) / 2 b}}{t \sqrt{t-1}} d t \\
& =\frac{\sqrt{a} e^{a / 2 b}}{b \sqrt{b}} \int_{1}^{\infty} \frac{e^{-a t / 2 b}}{t \sqrt{t-1}} d t \\
& =\frac{2 \pi \sqrt{a} e^{a / 2 b}}{b \sqrt{b}} Q(\sqrt{a / b}),
\end{aligned}
$$

where the last equality follows from Lemma D.1. The assertion follows from (D.6), (D.7), and (D.9).

\section{Appendix E: Derivation of Solutions (33) AND (37)}

\section{A. Derivation of (33)}

The Lagrangian associated with (32) is

$$
\begin{aligned}
& L\left(p_{1}, \cdots, p_{N}, \lambda, \mu_{1}, \cdots, \mu_{N}\right) \\
& =\sum_{i=1}^{N}\left\{\frac{1}{\alpha_{i}+\delta_{i}}-\frac{\sigma_{v} \sqrt{\pi}}{p_{i}\left(\alpha_{i}+\delta_{i}\right) \sqrt{2\left(\alpha_{i}+\delta_{i}\right)}}\right\} \\
& -\lambda\left[\sum_{i=1}^{N} p_{i}^{2}-P\right]+\sum_{i=1}^{N} \mu_{i} p_{i},
\end{aligned}
$$

and the resultant KKT conditions are

$$
\begin{array}{r}
\sqrt{\frac{\pi}{2}} \times \frac{\sigma_{v}}{p_{i}^{2} \sqrt{\left(\alpha_{i}+\delta_{i}\right)^{3}}}-2 \lambda p_{i}+\mu_{i}=0, \\
\lambda \geq 0, \mu_{i} \geq 0, \mu_{i} p_{i}=0, p_{i} \geq 0,1 \leq i \leq N .
\end{array}
$$

It can be inferred from (E.2) that $p_{i}>0$; this is because, if $p_{i}=0$, we must have $\mu_{i}=-\infty$ and the condition (E.3) is violated. This then implies $\mu_{i}>0$, thereby $\lambda>0$ (see (E.2)), meaning that the total power constraint is active. The solution (33) can be obtained by solving $p_{i}$ based on (E.2) and (E.3).

\section{B. Derivation of (37)}

The associated Lagraingian is

$$
\begin{aligned}
& L\left(p_{1}, \cdots, p_{N}, \lambda, \mu_{1}, \cdots, \mu_{N}\right) \\
& =\sum_{i=1}^{N} p_{i}^{2}- \\
& \quad \lambda\left[\sum_{i=1}^{N}\left\{\frac{1}{\alpha_{i}+\delta_{i}}-\frac{\sigma_{v} \sqrt{\pi}}{p_{i}\left(\alpha_{i}+\delta_{i}\right) \sqrt{2\left(\alpha_{i}+\delta_{i}\right)}}\right\}-\varepsilon^{-1}\right] \\
& -\sum_{i=1}^{N} \mu_{i} p_{i}
\end{aligned}
$$


and the resultant KKT conditions are

$$
\begin{gathered}
2 p_{i}-\lambda\left[\sqrt{\frac{\pi}{2}} \times \frac{\sigma_{v}}{p_{i}^{2} \sqrt{\left(\alpha_{i}+\delta_{i}\right)^{3}}}\right]-\mu_{i}=0, \\
\lambda\left[\sum_{i=1}^{N}\left\{\frac{1}{\alpha_{i}+\delta_{i}}-\frac{\sigma_{v} \sqrt{\pi}}{p_{i}\left(\alpha_{i}+\delta_{i}\right) \sqrt{2\left(\alpha_{i}+\delta_{i}\right)}}\right\}-\varepsilon^{-1}\right]=0,
\end{gathered}
$$

$$
\lambda \geq 0, \mu_{i} \geq 0, \mu_{i} p_{i}=0, p_{i} \geq 0,1 \leq i \leq N
$$

It can be inferred that $p_{i}>0$ since, if $p_{i}=0$, we must have $\mu_{i}=-\infty$ and the condition (E.7) is violated. Hence, we can solve $p_{i}$ from (E.5) as

$$
p_{i}=\frac{(\pi / 2)^{1 / 6} \sqrt[3]{\lambda \sigma_{v}}}{\sqrt{2\left(\alpha_{i}+\delta_{i}\right)}}
$$

Also, the condition $p_{i}>0$ also implies $\lambda>0$, thereby

$$
\sum_{i=1}^{N}\left\{\frac{1}{\alpha_{i}+\delta_{i}}-\frac{\sigma_{v} \sqrt{\pi}}{p_{i}\left(\alpha_{i}+\delta_{i}\right) \sqrt{2\left(\alpha_{i}+\delta_{i}\right)}}\right\}=\varepsilon^{-1} .
$$

The solution (37) can be directly obtained by solving for $p_{i}$ based on (E.9).

\section{ApPENDIX F: DERIVATION OF SOLUTION (51)}

To solve the problem, let us form the Lagrangian associated with (49) as

$$
\begin{aligned}
& L\left(P_{1}, \cdots, P_{N}, \lambda, \mu_{1}, \cdots, \mu_{N}\right) \\
& =\sum_{i=1}^{N} \frac{P_{i}}{\left(\alpha_{i}+\delta_{i}\right) P_{i}+\gamma_{i}}-\lambda\left[\sum_{i=1}^{N} P_{i}-P\right]+\sum_{i=1}^{N} \mu_{i} P_{i}
\end{aligned}
$$

the associated set of KKT conditions then reads

$$
\begin{gathered}
\frac{\gamma_{i}}{\left[\left(\alpha_{i}+\delta_{i}\right) P_{i}+\gamma_{i}\right]^{2}}-\lambda+\mu_{i}=0,1 \leq i \leq N, \\
\lambda\left[\sum_{i=1}^{N} P_{i}-P\right]=0,
\end{gathered}
$$

$$
\lambda \geq 0, \mu_{i} \geq 0, \mu_{i} P_{i}=0, P_{i} \geq 0,1 \leq i \leq N .
$$

By solving (F.2) we have

$$
P_{i}=\frac{\sqrt{\gamma_{i}}}{\left(\alpha_{i}+\delta_{i}\right) \sqrt{\lambda-\mu_{i}}}-\frac{\gamma_{i}}{\left(\alpha_{i}+\delta_{i}\right)},
$$

based on which $\lambda$ and $\mu_{i}$ 's should be determined to fulfill the desired constraints. Note that, if $\lambda=0$, equation (F.2) implies $\mu_{i}<0$ for all $1 \leq i \leq N$, which is impossible. Hence we must then have $\lambda \neq 0$, and the total power constraint in (F.3) is active. To find the solution, we sort the reciprocal of channel SNR $\gamma_{i}=\sigma_{v}^{2} / h_{i}^{2}$, s as $\gamma_{1} \geq \gamma_{2} \geq \cdots \geq \gamma_{N}$. With $f(K)$ defined in (50), we can show that if there exists $1 \leq K_{1} \leq N$ such that $f\left(K_{1}\right) \geq 1$, then $f\left(K_{1}+1\right) \geq 1$ (hence, such a $K_{1}$ is unique). By rearranging we have

$$
\begin{aligned}
& f\left(K_{1}+1\right) \\
& =\frac{P+\sum_{j=K_{1}+1}^{N} \frac{\gamma_{j}}{\left(\alpha_{j}+\delta_{j}\right)}}{\sqrt{\gamma_{K_{1}+1}} \sum_{j=K_{1}+1}^{N} \frac{\sqrt{\gamma_{j}}}{\left(\alpha_{j}+\delta_{j}\right)}} \\
& =\frac{P+\sum_{j=K_{1}}^{N} \frac{\gamma_{j}}{\left(\alpha_{j}+\delta_{j}\right)}-\frac{\gamma_{K_{1}}}{\left(\alpha_{K_{1}}+\delta_{K_{1}}\right)}}{\sqrt{\gamma_{K_{1}+1}}\left[\sum_{j=K_{1}}^{N} \frac{\sqrt{\gamma_{j}}}{\left(\alpha_{j}+\delta_{j}\right)}-\frac{\sqrt{\gamma_{K_{1}}}}{\left(\alpha_{K_{1}}+\delta_{K_{1}}\right)}\right]} \\
& \stackrel{P}{(a)} \frac{P+\sum_{j=K_{1}}^{N} \frac{\gamma_{j}}{\left(\alpha_{j}+\delta_{j}\right)}-\frac{\gamma_{K_{1}}}{\left(\alpha_{K_{1}}+\delta_{K_{1}}\right)}}{\sqrt{\gamma_{K_{1}}}\left[\sum_{j=K_{1}}^{N} \frac{\sqrt{\gamma_{j}}}{\left(\alpha_{j}+\delta_{j}\right)}-\frac{\sqrt{\gamma_{K_{1}}}}{\left(\alpha_{K_{1}}+\delta_{K_{1}}\right)}\right]} \\
& =\frac{P+\sum_{j=K_{1}}^{N} \frac{\gamma_{j}}{\left(\alpha_{j}+\delta_{j}\right)}-\frac{\gamma_{K_{1}}}{\left(\alpha_{K_{1}}+\delta_{K_{1}}\right)}}{\sqrt{\gamma_{K_{1}}} \sum_{j=K_{1}}^{N} \frac{\sqrt{\gamma_{j}}}{\left(\alpha_{j}+\delta_{j}\right)}-\frac{\gamma_{K_{1}}}{\left(\alpha_{K_{1}}+\delta_{K_{1}}\right)}} \\
& \stackrel{(b)}{=} \frac{\left[P+\sum_{j=K_{1}}^{N} \frac{\gamma_{j}}{\left(\alpha_{j}+\delta_{j}\right)}\right]\left[\sqrt{\gamma_{K_{1}}} \sum_{j=K_{1}}^{N} \frac{\sqrt{\gamma_{j}}}{\left(\alpha_{j}+\delta_{j}\right)}\right]^{-1}-\tau}{1-\tau} \\
& =\frac{f\left(K_{1}\right)-\tau}{1-\tau} \geq 1,
\end{aligned}
$$

where (a) follows since $\gamma_{K_{1}} \geq \gamma_{K_{1}+1}$, (b) holds by writing $\tau=\frac{\gamma_{K_{1}}}{\left(\alpha_{K_{1}}+\delta_{K_{1}}\right)}\left[\sqrt{\gamma_{K_{1}}} \sum_{j=K_{1}}^{N} \frac{\sqrt{\gamma_{j}}}{\left(\alpha_{j}+\delta_{j}\right)}\right]^{-1}$, and (c) results since $f\left(K_{1}\right) \geq 1$ by assumption. Solution (51) can then be obtained by using (F.3) and (F.5) together with the established property of $f(K)$.

\section{Appendix G: Derivation of Solution (54)}

The Lagrangian associated with (49) is

$$
\begin{aligned}
& L\left(P_{1}, \cdots, P_{N}, \lambda, \mu_{1}, \cdots, \mu_{N}\right) \\
& =\sum_{i=1}^{N} P_{i}-\lambda\left[\sum_{i=1}^{N} \frac{P_{i}}{\left(\alpha_{i}+\delta_{i}\right) P_{i}+\gamma_{i}}-\varepsilon^{-1}\right]-\sum_{i=1}^{N} \mu_{i} P_{i},
\end{aligned}
$$

which leads to the KKT conditions

$$
\begin{gathered}
1-\lambda\left\{\frac{\gamma_{i}}{\left[\left(\alpha_{i}+\delta_{i}\right) P_{i}+\gamma_{i}\right]^{2}}\right\}-\mu_{i}=0,1 \leq i \leq N \\
\lambda\left[\sum_{i=1}^{N} \frac{P_{i}}{\left(\alpha_{i}+\delta_{i}\right) P_{i}+\gamma_{i}}-\varepsilon^{-1}\right]
\end{gathered}
$$

$$
\lambda \geq 0, \mu_{i} \geq 0, \mu_{i} P_{i}=0, P_{i} \geq 0,1 \leq i \leq N
$$

If $\lambda=0$, then (G.2) implies $\mu_{i}>0$, and hence, $P_{i}=0$, for $1 \leq i \leq N$, that is, all sensors are shut off. This case should be precluded, and we must have $\lambda>0$, meaning that the distortion constraint is active. To derive (54), we sort $\gamma_{i}$ 's as $\gamma_{1} \geq \gamma_{2} \geq \cdots \geq \gamma_{N}$ and define the function $g(K)$ as in (53). We first show that that, if there exists $1 \leq K_{2} \leq N$ such that $g\left(K_{2}\right) \geq 1$, then $g\left(K_{2}+1\right) \geq 1$ (hence such a $K_{2}$ is 
unique). To see this, by rearranging we have

$$
\begin{aligned}
& g\left(K_{2}+1\right) \\
& =\frac{\sum_{j=K_{2}+1}^{N} \frac{\sqrt{\gamma_{j}}}{\left(\alpha_{j}+\delta_{j}\right)}}{\sqrt{\gamma_{K_{2}+1}}\left[\sum_{j=K_{2}+1}^{N} \frac{1}{\left(\alpha_{j}+\delta_{j}\right)}-\varepsilon^{-1}\right]} \\
& =\frac{\sum_{j=K_{2}}^{N} \frac{\sqrt{\gamma_{j}}}{\left(\alpha_{j}+\delta_{j}\right)}-\frac{\sqrt{\gamma_{2}}}{\left(\alpha_{K_{2}}+\delta_{K_{2}}\right)}}{\sqrt{\gamma_{K_{2}+1}}\left[\sum_{j=K_{2}}^{N} \frac{1}{\left(\alpha_{j}+\delta_{j}\right)}-\varepsilon^{-1}-\frac{1}{\left(\alpha_{K_{2}}+\delta_{K_{2}}\right)}\right]} \\
& \stackrel{(a)}{\geq} \frac{\sum_{j=K_{2}}^{N} \frac{\sqrt{\gamma_{j}}}{\left(\alpha_{j}+\delta_{j}\right)}-\frac{\sqrt{\gamma_{K_{2}}}}{\left(\alpha_{K_{2}}+\delta_{K_{2}}\right)}}{\sqrt{\gamma_{K_{2}}}\left[\sum_{j=K_{2}}^{N} \frac{1}{\left(\alpha_{j}+\delta_{j}\right)}-\varepsilon^{-1}-\frac{1}{\left(\alpha_{K_{2}}+\delta_{K_{2}}\right)}\right]} \\
& =\frac{\sum_{j=K_{2}}^{N} \frac{\sqrt{\gamma_{j}}}{\left(\alpha_{j}+\delta_{j}\right)}-\frac{\sqrt{\gamma_{K_{2}}}}{\left(\alpha_{K_{2}}+\delta_{K_{2}}\right)}}{\sqrt{\gamma_{K_{2}}}\left[\sum_{j=K_{2}}^{N} \frac{1}{\left(\alpha_{j}+\delta_{j}\right)}-\varepsilon^{-1}\right]-\frac{\sqrt{\gamma_{K_{2}}}}{\left(\alpha_{K_{2}}+\delta_{K_{2}}\right)}} \\
& \stackrel{(b)}{=} \frac{\left[\sum_{j=K_{2}}^{N} \frac{\sqrt{\gamma_{j}}}{\left(\alpha_{j}+\delta_{j}\right)}\right]\left[\sqrt{\gamma_{K_{2}}}\left(\sum_{j=K_{2}}^{N} \frac{1}{\left(\alpha_{j}+\delta_{j}\right)}-\varepsilon^{-1}\right)\right]^{-1}}{1-\lambda} \\
& -\frac{\lambda}{1-\lambda} \\
& =\frac{g\left(K_{2}\right)-\lambda}{1-\lambda} \geq 1,
\end{aligned}
$$

where (a) follows since $\gamma_{K_{2}} \geq \gamma_{K_{2}+1}$, (b) holds by writing $\lambda=\frac{1}{\left(\alpha_{K_{2}}+\delta_{K_{2}}\right)}\left[\sum_{j=K_{2}}^{N} \frac{1}{\left(\alpha_{j}+\delta_{j}\right)}-\varepsilon^{-1}\right]^{-1}$, and (c) results since $g\left(K_{2}\right) \geq 1$ by assumption. Solution (54) can then be obtained by using (G.3) and the established property of the function $g(K)$.

\section{REFERENCES}

[1] I. F. Akyildiz, W. Su, Y. Sankarasubramaniam, and E Cayirci, "A survey on sensor networks," IEEE Commun. Mag., vol. 40, no. 8, pp. 102-114, Aug. 2002.

[2] B. M. Sadler, "Fundamentals of energy-constrained sensor networks," IEEE Aerosp. Electron. Syst. Mag., vol. 20, no. 8, pp. 17-35, Aug. 2005.

[3] R. Viswanathan and P. K. Varshney, "Distributed detection with multiple sensors-part I: fundamentals," Proc. IEEE, vol. 85, no. 1, pp. 54-63, Jan. 1997.

[4] J. J. Xiao, A. Ribeiro, Z. Q. Luo, and G. B. Giannakis, "Distributed compression-estimation using wireless sensor networks," IEEE Signal Process. Mag., vol. 23, no. 4, pp. 27-41, July 2006.

[5] Q. Zhao, A. Swami, and L. Tong, "The interplay between signal processing and networking in sensor networks," IEEE Signal Process. Mag., vol. 23, no. 4, pp. 84-93, July 2006.

[6] A. Ribeiro and G. B. Giannakis, "Bandwidth-constrained distributed estimation for wireless sensor networks-part I: Gaussian case," IEEE Trans. Signal Process., vol. 54, no. 3, pp. 1131-1143, Mar. 2006.

[7] A. Ribeiro and G. B. Giannakis, "Bandwidth-constrained distributed estimation for wireless sensor networks-part II: unknown probability density function," IEEE Trans. Signal Process., vol. 54, no. 7, pp. 27842796, July 2006.

[8] X. Wu and Z. Tian, "Optimized data fusion in bandwidth and energy constrained sensor networks," in Proc. 2006 IEEE ICASSP, vol. 4, pp. $713-716$.

[9] T. Zhao and A. Nehorai, "Information-driven distributed maximum likelihood estiamtion based on Gaussian-Newton method in wireless sensor networks," IEEE Trans. Signal Process., vol. 55, no. 9, pp. 46694682, Sep. 2007.

[10] H. Li and J. Fang, "Distributed adaptive quantization and estimation for wireless sensor networks," IEEE Signal Process. Lett., vol. 14, pp. 669-672, Oct. 2007.

[11] J. Fang and H. Li, "Distributed adaptive quantization for wireless sensor networks: from delta modulation to maximum likelihood," IEEE Trans. Signal Process., vol. 56, no. 10, pp. 5246-5257, Oct. 2008.

[12] J. Fang and H. Li, "Hyperplane-based vector quantization for distributed estimation in wireless sensor networks," IEEE Trans. Inf. Theory, vol. 55, no. 12, pp. 5682-5699, Dec. 2009.
[13] A. Krasnopeev, J. J. Xiao, and Z. Q. Luo, "Minimum energy decentralized estimation in sensor network with correlated sensor noise," EURASIP J. Wireless Commun. Netw., vol. 4, pp. 473-482, 2005.

[14] J. J. Xiao, S. Cui, Z. Q. Luo, and A. J. Goldsmith, "Power scheduling of universal decentralized estimation in sensor networks," IEEE Trans. Signal Process., vol. 54, no. 2, pp. 413-422, Feb. 2006.

[15] Z. Q. Luo, "Universal decentralized estimation in a bandwidth constrained sensor networks," IEEE Trans. Inf. Theory, vol. 51, no. 6, pp. 2210-2219, June 2005.

[16] S. Cui, J. J. Xiao, A. J. Goldsmith, and H. V. Poor, "Estimation diversity and energy efficiency in distributed sensing," IEEE Trans. Signal Process., vol. 55, no. 9, pp. 4683-4696, Sep. 2007.

[17] J. J. Xiao, S. Cui, Z. Q. Luo, and A. J. Goldsmith, "Linear coherent decentralized estimation," IEEE Trans. Signal Process., vol. 56, no. 2, pp. 757-770, Feb. 2008.

[18] J. Y. Wu, Q. Z. Huang, and T. S. Lee, "Minimal energy decentralized estimation via exploiting the statistical knowledge of sensor noise variance," IEEE Trans. Signal Process., vol. 56, no. 5, pp. 2171-2176, May 2008.

[19] J. Y. Wu, Q. Z. Huang, and T. S. Lee, "Energy-constrained decentralized best-linear-unbiased estimation via partial sensor noise variance knowledge," IEEE Signal Process. Lett., vol. 15, pp. 33-36, 2008.

[20] H. Senol and C. Tepedelenlioglu, "Performance of distributed estimation over unknown parallel channels," IEEE Trans. Signal Process., vol. 56, no. 12, pp. 6057-6068, Dec. 2008.

[21] J. Li and G. AlRegib, "Function-based network lifetime for estimation in wireless sensor networks," IEEE Signal Process. Lett., vol. 15, pp. 533-536, 2008.

[22] J. Fang and H. Li, "Power constrained distributed estimation with cluster-based sensor cooperation," IEEE Trans. Wireless Commun., vol. 8, no. 7, pp. 3822-3832, July 2009.

[23] J. Fang and H. Li, "Power constrained distributed estimation with correlated sensor data," IEEE Trans. Signal Process., vol. 57, no. 8, pp. 3292-3297, Aug. 2009.

[24] E. Ertin, R. Moses, and L. Potter, "Network parameter estimation with detection failures," in Proc. 2004 IEEE ICASSP, vol. 2, pp. 273-276.

[25] V. Delouille, R. Neelamani, and R. G. Baraniuk, "Robust distributed estimation using the embedded subgraphs algorithms," IEEE Trans. Signal Process., vol. 54, no. 8, pp. 2998-3010, Aug. 2006.

[26] X. Qu, J. Zhou, E. Song, and Y. Zhu, "Minimax robust optimal estimation fusion in distributed multisensor systems with uncertainties," IEEE Signal Process. Lett., vol. 17, no. 9, pp. 811-814, Sep. 2010.

[27] B. Chen, R. Jiang, T. Kasetkasem, and P. K. Varshney, "Channel aware decision fusion in wireless sensor networks," IEEE Trans. Signal Process., vol. 52, no. 12, pp. 3454-3458, Dec. 2004.

[28] Y. Lin, B. Chen, and P. K. Varshney, "Decision fusion rules in multi-hop wireless sensor networks," IEEE Trans. Aerosp. Electron. Syst., vol. 41, no. 2, pp. 475-488, Apr. 2005.

[29] R. Niu, B. Chen, and P. K. Varshney, "Fusion of decisions transmitted over Rayleigh fading channels in wireless sensor networks," IEEE Trans. Signal Process., vol. 54, no. 3, pp. 1018-1027, Mar. 2006.

[30] I. Bar-David and P. Boukris, "Reciprocal mean-square error and signalto-noise ratio as distinct performance measures in below-threshold communication," IEEE Trans. Commun., vol. 20, no. 5, pp. 852-856, Oct. 1972.

[31] I. S. Gradshteyn and I. M. Ryzhik, Tables of Integrals, Series, and Products, 7th ed. Academic Press, 2007.

[32] L. Zhang, T. Cui, T. Ho, and X. D. Zhang, "Optimal quantization in energy-constrained sensor networks under imperfect transmissions," in Proc. 2009 Conf. Inf. Sciences Syst., pp. 613-617.

[33] M. H. Chaudhary and Luc Vandendorpe, "Power constrained linear estimation in wireless sensor networks with correlated data and digital modulation," IEEE Trans. Signal Process., vol. 60, no. 2, pp. 570-584, Feb. 2012

[34] S. M. Kay, Fundamentals of Statistical Signal Processing: Estimation Theory. Prentice-Hall PTR, 1993.

[35] S. M. Kay, Fundamentals of Statistical Signal Processing: Detection Theory. Prentice-Hall PTR, 1998.

[36] A. Leon-Garcia, Probability and Random Process for Electrical Engineering, 3rd ed. Addison-Wesley, 2009.

[37] M. K. Banavar, C. Tepedelenlioglu, and A. Spanias, "Estimation over fading channels with limited feedback using distributed sensing," IEEE Trans. Signal Process., vol. 58, no. 1, pp. 414-425, Jan. 2010.

[38] T. Wimalajeewa and S. Jayaweera, "Optimal power scheduling for correlated data fusion in wireless sensor networks via constrained PSO," IEEE Trans. Wireless Commun., vol. 7, no. 9, pp. 3608-3618, Sep. 2008. 
[39] I. Boussaid, P. Siarry, and M. Ahmed-Nacer, "Hybridizing biogeography-based optimization with differential evoluation for optimal power allocation in wireless sensor networks," IEEE Trans. Veh. Technol., vol. 60, no. 5, pp. 2347-2353, May 2011.

[40] T. M. Cover and J. A. Thomas, Elements of Information Theory, 2nd ed. Wiley-Interscience, 2006.

[41] M. K. Simon and M.-S. Alouini, Digital Communications over Fading Channels: A Unified Approach to Performance Analysis. John Wiley \& Sons, 2000.

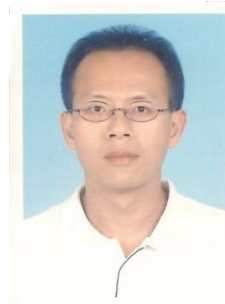

Jwo-Yuh Wu (M'04) received the B. S. degree in 1996, the M. S. degree in 1998, and the Ph. D. degree in 2002, all in electrical and control engineering from National Chiao Tung University, Taiwan. During 2003 and 2007, he was a post doctor research fellow in the Department of Communications Engineering, National Chiao Tung University, Taiwan. Since 2008, he has been a faculty member of the Department of Electrical and Computer Engineering, and the Institute of Communications Engineering, National Chiao Tung University, Taiwan, where he is currently an associate professor. His general research interests are in signal processing, wireless sensing and communications, control systems, linear algebra, and applied functional analysis.

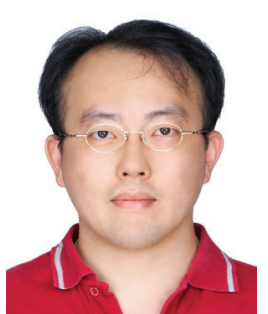

Tsang-Yi Wang (S'01-M'04) received the B.S. and M.S. degrees from National Sun Yat-sen University, Kaohsiung, Taiwan, in 1994 and 1996, respectively, and the Ph.D. degree in electrical engineering from Syracuse University, NY, in 2003. From 2004 to 2006, he was an Assistant Professor in the Graduate Institute of Communication Engineering at National Chi Nan University, Nantou, Taiwan. In February 2006, he joined the faculty of the Institute of Communications Engineering, National Sun Yatsen University, Kaohsiung, Taiwan, as an Assistant Professor, and in August 2008, he became an Associate Professor. He was promoted to Professor in August 2012. He has served as a Reviewer Editor of the Journal of Wireless Communications and Mobile Computing. His research mainly focuses on distributed detection and estimation with applications in wireless communications and wireless sensor networks.

Dr. Wang received the 2008 Best Paper Award for Young Scholars awarded from the IEEE Information Society Taipei Chapter and the IEEE Communications Society Taipei/Tainan Chapter. 